\title{
Philosophiques
}

\section{La géométrie : clef du réel? Pensée de l'espace et philosophie des mathématiques}

\section{Luciano Boi}

Volume 24, numéro 2, automne 1997

URI : https://id.erudit.org/iderudit/027460ar

DOI : https://doi.org/10.7202/027460ar

Aller au sommaire du numéro

Éditeur(s)

Société de philosophie du Québec

ISSN

0316-2923 (imprimé)

1492-1391 (numérique)

Découvrir la revue

Citer cet article

Boi, L. (1997). La géométrie : clef du réel? Pensée de l'espace et philosophie des mathématiques. Philosophiques, 24(2), 389-430.

https://doi.org/10.7202/027460ar d'utilisation que vous pouvez consulter en ligne.

https://apropos.erudit.org/fr/usagers/politique-dutilisation/ 


\title{
LA GÉOMÉ TRIE : CLEF DU RÉEL ? PENSÉE de l'ESPACE E PHILOSOPHIE dES MATHÉMATIQUES
}

\author{
PAR \\ LUCIANO BOI
}

Les articles d'Alain Michel et de Jean Petitot abordent un certain nombre de questions philosophiques importantes concernant les développements des mathématiques et de la physique modernes, que nous avons analysés dans l'ouvrage Le problème mathématique de l'espace. Une quête de l'intelligible (Springer, 1995). Après avoir présenté ceux qui lui paraissent, à juste titre, les principaux thèmes historiques et épistémologiques de l'ouvrage, A. Michel passe à discuter certaines de nos thèses philosophiques. Il apporte des éclaircissements notables sur plusieurs points difficiles et controversés, en particulier, le rapport entre les espaces sensible, mathématique et physique, l'interprétation du conventionnalisme géométrique de Poincaré et le statut des concepts mathématiques. Il adresse également quelques critiques à certaines de nos thèses, qui touchent essentiellement à la question épistémologique de savoir quelle est la nature des objets mathématiques. Les considérations qui suivent sont en grande partie consacrées à développer, sur certains points, les thèses en question. Par là, nous espérons parvenir à une plus grande clarté et à justifier davantage notre position.

Quelques mots sur l'article de Jean Petitot qui, à vrai dire, ne porte pas sur notre ouvrage, mais plutôt sur ses propres travaux, et où il réaffirme des thèses soutenues ailleurs. En ce sens, son article ne demande pas à être commenté ici. Cependant, puisque Petitot touche indirectement à des questions précises que nous avons largement analysées dans le livre, nous reviendrons sur certaines d'entre elles. Quoique toute son argumentation s'appuie sur des problèmes qui sont très techniques et difficiles, mais qui sont par ailleurs d'un grand intérêt, son raisonnement philosophique peut se résumer en une seule thèse. Les développements des sciences modernes (en particulier des mathématiques et de la physique) depuis la découverte des géométries non euclidiennes et l'élaboration par Lagrange et Hamilton d'une mécanique analytique nouvelle, n'auraient fait que confirmer le bien-fondé et la validité de la conception transcendantale kantienne des sciences, à la condition toutefois d'entendre celle-ci comme une "doctrine de la constitution des objectivités "ou comme "théorie des conditions de possibilité de l'expérience »et non pas comme une doctrine des facultés de la 
raison et de l'intellect. Mais il n'est pas du tout certain que ces deux aspects de la conception transcendantale se trouvent séparés chez Kant ${ }^{1}$. Nous discuterons ici certains aspects que cette thèse comporte, en tant qu'ils se rattachent aux thèmes et aux idées mathématiques et philosophiques dont nous avons traité dans l'ouvrage.

Pour les raisons que nous venons de rappeler, ce que nous nous proposons de faire ici n'est pas tellement de répliquer aux arguments et éventuellement aux critiques d'A. Michel et de J. Petitot, mais plutôt d'accepter un dialogue à trois sur des questions qui touchent aux sujets abordés dans notre ouvrage, mais dont la portée va bien au-delà et qui intéressent au premier chef les perspectives actuelles de la philosophie des sciences.

Avant d'en venir aux propos essentiels de notre ouvrage, nous croyons utile de faire quelques précisions et distinctions en quelque sorte préliminaires. Lorsqu'on parle, d'un point de vue épistémologique, du problème mathématique de l'espace, il importe en premier lieu de distinguer entre l'espace perceptif ${ }^{2}$, l'espace mathématique et l'espace physique. Chacun de ces espaces peut présenter des propriétés fort différentes et, par conséquent, des significations différentes. Il convient ensuite de distinguer au moins deux niveaux pour chacun de ces espaces, ce qui devrait permettre d'établir un certain nombre de correspondances (en fait, d'applications) à la fois mathématiques et physiques. Par "espace perceptif "il faut ainsi entendre aussi bien l'espace sensible (psycho-physiologique) que l'espace intuitif ; ce dernier est fondé sur un certain continuum qualitatif apparemment amorphe mais d'où, grâce à des schèmes représentationnels de nature géométrique (et non pas computationnelle) et à des esquisses synthétiques, émergent les Gestalt et les différentes qualités des objets. Or, l'espace intuitif peut être vu en quelque sorte comme un prolongement du premier, sans qu'jl puisse pour autant y être identifié ou réduit (voir plus loin pour des considérations à ce sujet). D'autre part, il est clair que, pour autant qu'il permet de donner une existence (idéale) précise à des objets qui n'existent que sous forme de potentialités, l'espace mathématique partage quelque chose d'essentiel avec l'espace intuitif, à moins de le concevoir (comme tous

1. Pour une analyse très intéressante de la conception kantienne des sciences et en particulier du statut des mathématiques dans la physique, voir P. Kerszberg, a Natural Versus Transcendental Philosoplyy ", Graduate Faculty Philosophy Journal, Vol. 18, n² 2 (1995), p. 17. 61.

2 L'espace perceptif lui-mème est formé de différents niveaux. On distinguera en bref : (i) le niveau pré-spatial (les différentes séries organisées d'impressions sensorielles) ;

(ii) le niveau spatial objectif (tridimensionnel) ; c'est le niveau où se constituent les formes spatiales proprement dites et ce, grâce au fait que nous sommes capables de discriminer un certain nombre de paramètres importants (doués d'une certaine pérennité) et de négliger ceux qui apparaissent aléatoires et/ou accidentels ;

(iii) le niveau symbolique etou scientifique, qui prolonge la perception au-delà de ses limites naturelles et qui apparait fondamental en vue de connaitre les structures invisibles (microscopiques) ou les phénomènes qui sont à trop grande échelle pour pouvoir ètre perçus (le comportement des astres). 
les objets qu'on peut, en théorie, y définir) soit comme un produit de notre activité cérébrale (cognitivisme), soit comme un univers linguistique formé d'entités fantasmagoriques (nominalisme). Mais il est vrai par ailleurs que l'espace mathématique, en tant que pure idéalisation ou en tant que modèle servant à représenter et à expliquer les phénomènes physiques, peut présenter des propriétés qui sont tout à fait différentes de celles que l'on accorde à l'espace intuitif. Il suffit de penser, par exemple, aux propriétés géométriques "bizarres "que caractérisent certains espaces topologiques symplectiques, ou bien encore, à la géométrie exotique de l'espace-temps à l'échelle quantique.

Mais revenons à notre réflexion sur les différents espaces. Pour ce qui est de l'espace physique, il est fondamental de distinguer au moins entre l'espace physique réel (où il est question des propriétés et des lois physiques des corps définies par des relations géométriques abstraites) et l'espace (en fait, il faudrait dire les espaces) de la géométrie physique, qui est un modèle mathématique abstrait. On a ainsi l'espace de Minkowski (caractérisé par une géométrie hyperbolique), l'espace d'Einstein (doué d'une géométrie pseudoriemannienne ou, dans la version de Penrose, d'une géométrie projective complexe), l'espace de Weyl (dont la structure géométrique est affine etou conforme), etc. La géométrie physique se situe ainsi à un niveau intermédiaire entre la géométrie mathématique proprement dite et la géométrie du monde réel. Le lien entre les deux est assuré par les groupes, qui peuvent être de nature fort différente (voir plus loin) ; plus précisément, ce sont eux qui relient les symétries des espaces abstraits aux symétries des théories physiques. En effet, dans les théories physiques récentes toutes les forces sont comprises au moyen de théories mathématiques qui ont la même forme : les théories de jauge non abéliennes avec des symétries locales. Ainsi, les théories mathématico-physiques ramènent les propriétés des forces aux symétries de la théorie d'une part, et aux symétries de la nature d'autre part. Ces symétries sont en général de nature spatiale ou géométrique, mais il existe également des symétries non géométriques comme, par exemplc, la symétrie de la charge. Les symétries du premier type se laissent définir mathématiquement comme des invariants. Il y a lieu de faire enfin une dernière distinction, entre la géométrie mathématique (qui n'obéit en principe à aucune contrainte physique), la géométrie de la physique théorique (qui élabore des modèles mathématiques pour l'explication des phénomènes) et la géométrie réelle, que l'on suppose être la géométrie à laquelle obéissent les lois du monde physique. À ce propos, de même qu'on peut concevoir plusieurs géométries mathématiques ainsi que plusieurs modèles théoriques de géométrie physique, de même, il peut y avoir plusieurs géométries réelles différentes. Par exemple, la géométrie du monde physique peut être tout à la fois localement non euclidienne bien que globalement euclidienne, et inversement, elle peut être localement euclidienne quoique globalement non euclidienne. 
Une telle relation entre les types mathématiques de géométries et les modèles physiques de géométrie, n'est pas le fruit d'un hasard. En fait, l'exploration puis la formalisation des propriétés mathématiques de chaque géométrie conceptuellement possible sont, en général, nécessaires à la compréhension des propriétés physiques correspondantes et elles permettent d'en déterminer en quelque sorte les conditions possibles d'existence. En ce sens, on peut dire que tout modèle mathématique de géométrie est un " univers virtuel » au sein duquel une géométrie physique peut passer d'un état de possibilité à un autre d'existence effective. La théorie de la supersymétrie ou la théorie des nœuds offrent de magnifiques exemples d'un tel processus. D'une manière générale, il nous paraît possible d'établir une première grande classification des géométries physiques. 1) La géométrie à l'échelle humaine (corps solides et courtes distances) est approximativement euclidienne; il y a cependant des situations pour lesquelles la géométrie diverge de manière sensible de l'euclidienne, c'est le cas, par exemple, de la géométrie du système visuel ${ }^{3}$. 2) La géométrie spatiale à large échelle (cosmologie) semble être en accord avec la géométrie de Lobatchevsky. En tout cas, toutes les expériences faites jusqu'à maintenant pour vérifier la relativité générale montrent que la structure géométrique de notre espace physique est courbe et non pas plane. Ce qui prouve, d'ailleurs, que toutes les géométries ne sont pas entre elles équivalentes, et que les raisons qui nous en font préférer une par rapport à telle ou à telle autre ne reposent pas sur un critère subjectif, comme le pensait Poincaré, mais sur des " faits " mathématiques et physiques bien précis. Rien n'empêche cependant que l'on ait à élargir et même à modifier profondément le modèle mathématique de géométrie physique accepté, surtout lorsqu'il s'agit de rendre compte d'un certain nombre de "faits "physiques nouvellement découverts ou des anomalies, par exemple, topologiques. C'est bien ce qui s'est produit dans le cas de la relativité générale, à la suite de la découverte des singularités dans la structure de l'espace-temps et des trous noirs. En fait, il semblerait nécessaire d'adopter un nouveau point de vue en ce qui concerne la conception que nous nous sommes faite jusqu'à maintenant de la théorie einsteinnienne de la géométrie de l'espace-temps, qui serait à fonder sur l'étude de la structure intrinsèque des bords de domaines complexes, et dont l'exemple le plus significatif est celui d'une variété complexe qui ne peut pas être localement étendue au-delà de ses bords $C^{\infty}$ différentiables et lisses. Ainsi, le fait que la théorie de la relativité générale soit fondée sur un modèle géométrique d'espace-temps pseudo-riemannien (une certaine variété $M$ différentiable à quatre dimensions et douée d'une métrique indéfinie $g$ )

3. Pour des considérations approfondies sur ce sujet, voir L. Boi, Géométrie et Perception. Modeles mathématiques et interprétations philosophiques de la perception spatiale, ouvrage à paraître.

4. Voir R. Penrose, * Physical Space-Time and Nonrealizable CR-Structures *, Proceedings of Symposia in Pure Mathematics, American Mathematical Society, Vol. 39 (1983), Part 1, p. 401 422. 
n'est guère arbitraire, mais cela tient à ce qu'un tel modèle permet d'interpréter de la façon la plus complète et cohérente possible les phénomènes physiques à cette échelle. Autrement dit, les concepts mathématiques appartenant à un tel modèle sont ceux qui reflètent et expliquent le mieux les événements du monde physique (bien que ces mêmes événements soient des " possibilités "ou des idéalisations) ${ }^{5}$. 3) La géométrie de l'infiniment petit (le monde de la microphysique) semble être, du moins jusqu'à une certaine approximation, non euclidienne mais dans un sens tout à fait non classique. En fait, il est très difficile pour le moment de dire à quoi ressemble la géométrie quantique. La seule chose qui apparaît certaine, c'est qu'elle est incompatible avec toutes les autres géométries connues de nos jours, à commencer par la géométrie euclidienne. Il semblerait d'abord possible que le modèle d'espace-temps usuel, qui est à la base des théories relativistes, perde complètement sa validité dans le monde quantique. La géométrie quantique n'a, à vrai dire, de sens que dans un espace de dimension très élevée (en fait infinie), et dont la structure topologique semble n'avoir rien en commun avec la structure d'une variété riemannienne ou pseudo-riemannienne différentiable et lisse.

Pour terminer ces considérations, disons que certaines affirmations d'A. Michel et de J. Petitot méritent d'être particulièrement soulignées, et qu'elles sont susceptibles de développements ultérieurs. Ajoutons que nous sommes fondamentalement d'accord avec la plupart d'entre elles, à quelques exceptions près, mais qui sont toutefois importantes, comme on le verra par la suite. D'abord, résumons leurs thèses.

1) Selon A. Michel, une lecture correcte de la conception transcendantale kantienne doit rendre justice au philosophe d'avoir bien compris qu'une géométrie indépendante de l'intuition peut bien être conçue du moment que l'on distingue la constitution de l'intuition de l'espace comme objet de l'intuition, de cette même intuition. En d'autres termes, Kant aurait compris " que la constitution formelle est requise comme condition préalable de la constitution de l'intuition empirique. Elle peut donc se constituer [...] comme objet d'une science indépendante, tout en étant comme condition, forme du monde. Elle est une sorte de pré-objet à l'égard de l'objet de l'expérience qu'elle rend possible, et non point un objet superposé à lui. La mathématique n'est donc plus subordonnée à la physique dans son existence, mais seulement dans sa valeur objective "(nous soulignons). Cependant Kant, selon A. Michel, n'aurait pas su tirer toutes les conséquences nécessaires d'une telle réflexion, car le problème principal est en fait a celui de savoir si la spatialité peut être objet d'une pensée en dehors du schème qui l'incarne ». C'est-à-dire qu'il importe, à ce moment-là, de distinguer (pour les réconcilier éventuellement par la suite) entre « la

5. Voir R. Geroch, « General Relativity , Proceedings of Symposia in Pure Mathematics, American Mathematical Society, Vol. 27 (1975), p. 401-414. 
spatialité comme condition (de représentation d'objets) et la spatialité comme objet de pensée. Si l'on parvient à préciser la conception d'un espace-objet, et non simplement d'un espace-condition, on pourra du même coup renouveler la signification du kantisme ". Pour cela, "il faudrait toujours corréler l'espace avec des systèmes opératoires : la spatialité traduirait le schématisme kantien, mais un schématisme non autonome, lié à des systèmes d'opérations. L'espace serait pensable comme un méta-objet : un objet plus général et plus abstrait, ou un objet-forme, qui serait lui-même condition de représentation d'objets, de différents objets spécifiques». En effet, si l'on regarde (pour ne citer qu'un exemple) les développements extraordinaires récents de la géométrie non commutative (dus notamment à $\mathrm{A}$. Connes), faits essentiellement en vue de fournir un cadre mathématique cohérent pour l'ensemble des pliénomènes quantiques, on s'aperçoit que cette géométrie est en fait une théorie mathématique autonome et puissante qui a permis, entre autres, un élargissement considérable du concept même d'espace, en particulier grâce à l'introduction de toute une nouvelle famille de structures algébriques, d'un ensemble d'opérations qui leur est associé et d'un nouveau type de calcul qui en découle. De cette manière, le cadre classique de la géométrie infinitésimale (avec des variables réelles ou complexes), qui a servi de fondement à toutes les théories physiques relativistes, se trouve remplacé par une géométrie quantique fondée sur un nouvel objet mathématique, à savoir des opérateurs définis sur l'espace d'Hilbert. Or, s’il est vrai que cette nouvelle géométrie, d'un point de vue purement mathématique, se suffit à elle-même, il est tout aussi vrai que sa signification profonde doit pouvoir provenir de ce qu'elle est apte à foumir une interprétation effective (ici effective doit s'entendre au sens de non conventionnelle et/ou non arbitraire, et non pas au sens de réelle) d'un domaine de phénomènes physiques ou mème d'une ou plusieurs théories physiques, comme il semblerait bien être le cas ${ }^{6}$. C'est dire qu'il n'y a pas de théorie mathématique signifiante qui ne soit pas, en principe, susceptible d'une interprétation en dehors de son propre domaine d'objets abstraits, que ce soit par des modèles intuitifs, physiques ou autres. On voit par là que le théorème d'incomplétude de Gödel est susceptible, en quelque sorte, d'une extension à l'ensemble des mathématiques.

2) À propos de Kant, Petitot propose de disjoindre deux dimensions de sa conception transcendantale (chose que Kant n'aurait pas vraiment faite), à savoir la dimension constitutive (la mathématique comme organon de l'expérience) et la dimension cognitive (théorie des facultés). La dimension constitutive revient à admettre des principes prescriptifs de la réalité physique, et elle concerne le problème des conditions de possibilité de l'expérience. Dans les termes de Petitot, “ conception constitutive » veut dire

6. Voir A. Connes, "Gravity coupled with matter and the foundation of non commutative geometry ^, Preprint, Institut des Hautes Études Scientiliques (Bures-sur-Yvette), mars 1996. 
détermination mathématique a priori des phénomènes physiques; en fait plus que cela: les phénomènes physiques ne sont rien de plus que les concepts mathématiques qui servent à les découvrir. L'exemple le plus décisif qui prouverait la "vérité " d'une telle assertion philosophique serait, selon lui, le rôle déterminant des symétries en physique.

Cette réduction des contenus des théories physiques aux concepts mathématiques conduit évidemment, entre autres, au refus de toute signification ontologique (théorie des substances) pour les entités physiques. Mais une telle conclusion n'est pas sans comporter des conséquences fâcheuses, aussi bien sur le plan scientifique que philosophique? Premièrement, elle nie que les théories physiques possèdent des contenus physiques propres, alors qu'en réalité, pour beaucoup d'objets de ces mêmes théories, il y a lieu de reconnaître une signification géométrique et une signification physique, qui ne se recouvrent pas forcément. Comme exemple, nous mentionnerions ici le fait qu'il existe des symétries géométriques (spatiales) et des symétries (dynamiques) qui ne sont pas nécessairement de nature géométrique. Ensuite, le fait qu'il existe des différences importantes entre les symétries externes (c'est-à-dire les groupes de Lorentz, de Poincaré et le groupe conforme), valables pour le cas de la masse au repos nulle, et les s)métries internes (les groupes $\mathrm{SU}(2)$ ou $(\mathrm{SU}(3)$ ) pour certaines propriétés des particules élémentaires. En fait, lorsque les théories quantiques (non linéaires) essayent d'incorporer le champ électromagnétique de Maxwell pour expliquer les particules élémentaires, ces deux types de symétries, de même que celui qui est responsable de la covariance (qui devrait permettre de coupler certaines propriétés quantiques des particules élémentaires à la gravitation), sont requis. Le problème est alors celui d'arriver à concevoir un espace courbe qui possède un certain type de structure topologique et qui soit invariant vis-à-vis d'un groupe dont la structure est beaucoup plus riche, et où il devient possible ainsi de rendre compatibles les trois types de symétries mentionnés.

Un autre exemple fondamental concerne la relation entre le potentiel de jauge et le champ de jauge, qui est à reconduire à la relation entre la connexion et la courbure. À ce propos, on peut observer qu'une connexion, qui est un objet mathématiquement bien défini, est plus primitive que la courbure. On peut, par conséquent, considérer le potentiel de jauge comme étant plus primitif que le champ de jauge. En fait, dans l'électromagnétisme, on peut montrer par des expériences physiques que le champ peut être identiquement nul, alors que des effets physiques peuvent encore être détectés. Ceux-ci sont dus au fait que le transport parallèle doit être non

7. Elle rappelle, à certains égards, le rêve logiciste de vouloir réduire les contenus des mathématiques à une langue logique parfaite. Encore plus réductif nous apparaît un certain point de vue cognitiviste qui prétend ramener la perception, l'intuition et la conscience à un petit nombre de mécanismes plus ou moins complexes de notre activité cérébrale (ou système neuronal). 
trivial dans le cas où la région de l'espace n'est pas simplement connexe. Le fait que la courbure s'annule, donne l'information nécessaire sur le transport parallèle le long des chemins (géodésiques) fermés. Dans le langage de la physique, le transport parallèle est généralement décrit en termes d'un facteur de phase non intégrable. Or, la propriété de la non-intégrabilité se réfère localement à l'existence d'un champ non nul, tandis qu'à grande échelle cette même propriété est de nature topologique et elle peut survenir même dans le cas de champs nuls. Classiquement, le concept de potentiel a été introduit comme un artifice mathématique pour simplifier les équations de champ et le caractère en quelque sorte arbitraire de la jauge dans le choix du potentiel indiquait que ce dernier n'avait pas de véritable signification physique. Mais, d'un point de vue géométrique, on peut en fait montrer qu'une telle interprétation n'est pas satisfaisantc. La connexion est un objet géométrique et le potentiel doit alors être considéré comme étant de nature physique. C'est le choix de la jauge grâce auquel on décrit le potentiel qui n’a pas de signification physique, ce qui correspond au fait que l'espace fibré (géométrique) qui " porte "la connexion ne possède pas de sections horizontales ${ }^{8}$.

\section{Quelques brefs rappels de l'ourrage}

Rappelons maintenant les principales thèses de l'ouvrage (nous y reviendrons par la suite).

- Le ncud de ce que nous avons appelé a la question épistémologique de l'espace " touche en fait au point suivant : il s'agit d'expliquer la double nature de l'espace, à savoir le fait qu'il est à la fois concept formel (ou intuition formalisante) constitutif de la géométrie - pensons au concept de variété ou de groupe - et principe d'intelligibilité et d'engendrement même des phénomènes (l'espace est, cn l'occurrence, encore un modèle abstrait mais qui aurait également une portée réelle et la fonction d'une intuition remplissante).

- Il importe de différencier le caractère apriorique de la perception spatiale de la réalité externe, de la forme a priori dans laquelle sont énoncées les propositions de la géométrie. Il s'agit de deux niveaux essentiellement différents de la question épistémologique de l'espace, qu'on a souvent confondus pour la seule raison qu'on n'avait pas encore fait la distinction entre les espaces sensible, mathématique et physique.

- D'un point de vue mathématique, on ne peut plus parler d'espace unique (comme la découverte des géométries non euclidiennes l'a définitivement montré) : il y a autant d'espaces qu'il y a de géométries, chacun d'eux pouvant être considéré comme un domaine d'êtres abstraits ou comme une forme d'idéalisation. Les contenus de tels espaces sont a priori, car

8. Voir M. Atiyah, Geometry of Yang-Mills Fields, Lezioni Fermiane, Accademia Nazionale dei Lincei, Scuola Normale Superiore, Pise, 1979. 
il devient possible, à partir de quelques concepts fondamentaux, de développer formellement les structures géométriques caractérisant ces espaces. Lesdits concepts sont constitutifs pour un domaine d'êtres géométriques. Poincaré et Husserl ont, entre autres, mis en évidence ce fait important selon des perspectives différentes. Mais la question de savoir à quelles conditions un espace peut être support d'une ou même de plusieurs géométries intéressantes, demeure toujours en grande partie sans réponse.

- Les concepts géométriques n'ont pas seulement un statut formel, puisqu'ils peuvent également exprimer à la fois des contenus mathématiques non formalisables (même la logique mathématique en fournit des exemples, it suffit de penser au théorème de Gödel), extra-formels (le continu) et physiques (la courbure). En effet, les entités géométriques idéales et leurs propriétés sont à même de déterminer les formes d'objets réels. On dira donc, en ce sens, qu'ils s'impliquent dans les phénomènes se déroulant dans l'espace physique, au lieu de tout simplement s'y appliquer. De ce point de vue, l'espace et ses propriétés géométriques (de même que sa topologie) ne peuvent pas être a priori conçus séparément du contenu matériel de ce même espace : les propriétés physiques des corps agissent sur leurs propriétés géométriques, et réciproquement. Dit en des termes plus actuels, un champ (électromagnétique ou gravitationnel) est considéré comme une connexion sur un fibré ; de sorte que la matière impose des conditions sur la courbure de cette connexion, et la connexion force la matière à se propager par " transport parallèle " le long des lignes d'univers. La notion de transport parallèle, qu'il revient à Levi-Civita puis à Weyl et à Cartan d'avoir élaborée dans le cadre du développement de la géométrie riemannienne, est devenue un concept fondamental de la physique, ce qui donne une idée de sa puissance explicative.

- À la lumière des remarques précédentes, on peut affirmer que le point de vue de ccrtains philosophes néo-kantiens comme Natorp et Cassirer, le conventionnalisme géométrique de Poincaré, la phénoménologie de Husserl, Becker et Weyl et la conception structuraliste des mathématiques des philosophes Gonseth, Lautman et Cavaillès se révèlent ètre des tentatives de révision profonde de la conception kantienne de l'espace et de la géométrie, et en constituent un développement essentiel. Ainsi, le thème du « synthétique a priori ne nous paraît pas dépassé, mais comme ayant changé de niveau et de signification. Avec Gauss, il passe du niveau métrique global (tel chez Euclide) au niveau métrique local (infinitésimal) incarné par le $d s^{2}$. Avec Riemann, il connaîtra un nouveau changement en passant du niveau métrique au niveau différentiable, alors que la métrique deviendra un fait conventionnel d'une part, et empirique d'autre part. Avec Poincaré, on verra renaître la composante synthétique a priori de la géométrie dans le rôle joué par le concept de groupe. Enfin, avec Weyl et Cartan, le synthétique a priori passe au niveau affine du transport parallèle et de la connexion, ces 
concepts étant vus comme des objets constitutifs de la géométrie de la variété et de l'espace.

- L’idée directrice de notre ouvrage est que l'espace est un principe d'intelligibilité formel et réel. En d'autres termes, les concepts spatiaux seraient des modèles géométriques (des idéalisations) qui serviraient notamment de constructions mentales intermédiaires entre la mathématique et la physique. C'est au fond dans ces constructions mentales que résiderait la signification mathématique de la physique. La géométrie différentielle riemannienne et non riemannienne et la théorie des groupes en sont des exemples extrêmement importants. Un concept géométrique n'est pas seulement une pure et libre création intellectuelle, mais encore, considéré dans sa relation aux phénomènes physiques (dynamiques), il est constitutif de ces mêmes phénomènes. Il faudrait alors dire que "le réel est géométrique ", plutôt que d'affirmer que " la géométrie est réelle ou empirique ".

\section{Le gémétrique (le virtuel) et le physique (l'actuel) sont dans la méme connexion mais sur des plans différ ents}

Dans cet article, nous aimerions faire quelques considérations sur certaines questions fondamentales concernant la nature de l'espace en mathématiques et en physique théorique pour en arriver à montrer que l'espace est en fait une prégnance à la fois objective et subjective " universelle " et que, de par ce fait même, il se trouve à la racine de toute compréhension que l'homme peut avoir du monde qui l'entoure tout comme de l'univers macroscopique et microscopique. Son étude soulève un certain nombre de problèmes épistémologiques cruciaux. Un de ceux-ci, qui cst peut-être le plus général, consiste à savoir quel est le statut des objets et des idées mathématiques. L'autre problème que l'on pourrait mentionner, à savoir celui des rapports entre philosophie et mathématique (que nous avons abordé surtout en relation aux critiques que Husserl a adressées aux conceptions de Riemann), s'y rattache plus ou moins directement.

Dans son étude, $A$. Michel cherche à élucider le premier de ces problèmes en y apportant quelques éléments nouveaux. À ce sujet, nos analyses semblent converger sur des points essentiels, sauf en ce qui concerne l'interprétation que nous avons donnée de la thèse de Husserl affirmant la primauté logique et phénoménologique de la géométrie euclidienne, thèse que nous avons critiquée à notre tour, mais qui en même temps nous a semblée être fondée sur des raisons mathématiques et philosophiques profondes (voir infra). Selon A. Michel, en revanche, cette incompréhension de Husserl à l'égard des conceptions de Riemann "était inévitable, déjà inscrite dans son plus vieux souci : celui de réassigner à la philosophie un projet radical, selon les Méditations cartésiennes, que celui de Descartes. Cavaillès a montré la présence, dans la Crisis, du thème de l'utopie de l'archéologie fondatrice, du mythe du retour aux "évidences primordiales": l'histoire n’étant pas en mesure de révéler les sens 
authentiques que dans la mesure où elle permet de retrouver les liens perdus ".

Nos positions divergent de façon plus profonde quant à la thèse selon laquelle les objets mathématiques auraient essentiellement un caractère qualitatif, spatial et structural. Précisons d'abord qu'en affirmant ceci, nous avions à l'esprit surtout les développements de certains domaines selon nous cruciaux des mathématiques, comme la géométrie et la topologie différentielle, la théorie des groupes, la théorie (topologique) des systènıes dynamiques, la physique mathématique, etc., alors que nous n'avions pas vraiment considéré d'autres domaines, certes importants, tels que la théoric des nombres. l'algèbre et la théorie des catégories. On pourrait d'ailleurs remarquer à ce propos que là aussi on parle (au moins depuis Minkowski) de " théorie géonétrique des nombres " et de "géométrie algébrique ". Mais notre thèse. il est vrai, est avant tout philosophique et elle demande à être précisée. Ce que nous entendions par cela peut être éclairé en considérant brièvement le concept de groupe, un des plus fondamentaux qui soient en mathématiques. On sait que, dans un certain sens, la définition d'une géométrie revient à se donner un groupe de transformations que l'on fait opérer sur un espace donné. En fait, l'espace peut être alors identifié à l'ensemble des transformations, les rotations dans le cas euclidien, qui laissent invariante une certaine forme (fonction) fondamentale, et dont il pourra hériter certaines propriétés essentielles : l'homogénéité, la transitivité, une symétricité locale ou globale. En ce sens, l'espace peut donc être engendré par l'action du groupe, qui peut être considéré comme l'objet primordial, alors que l'espace pourra être vu comme un objet (mathématique) dérivé.

Il faut en plus voir que ces transformations appartenant au groupe possèdent une structure algébrique (les algèbres de Lie par exemple; un groupe peut présenter également des propriétés arithmétiques et topologiques fondamentales : ce sont des structures supplémentaires qui s’ajoutent à la structure algébrique en l'enrichissant). Ces structures correspondent, dans un certain sens, à ce qu'A. Michel appelle un "système d'opérations formelles ». Toutefois ces opérations et règles formelles, qui font partie de la structure algébrique mais qui ne sont pas la structure algébrique elle-même, n'ont pas uniquement une valeur symbolique, car elles correspondent, dans la plupart des cas, à des propriétés géométriques de l'espace considéré, et elles peuvent également s'interpréter en termes de propriétés des entités physiques d'une certaine théorie.

Enfin, il importe de comprendre que l'objet mathématique groupe présente des propriétés qualitatives fondamentales et ce, dans un double sens : mathématique et physique (dynamique). Il suffit de penser, par exemple, au fait qu'une certaine classe d'équations différentielles à dérivées partielles, dont Poincaré en a, le premier, étudié le comportement et découvert l'importance pour l'explication des phénomènes de la mécanique 
céleste et, plus généralement, des systèmes dynamiques, présente des propriétés qualitatives fondamentales concernant la classification topologique d'un certain type de courbes et de surfaces ainsi que la connaissance de leurs propriétés globales. Or, ces propriétés correspondent en réalité à la donnée d'un groupe topologique dont les transformations conservent les relations conformes et continues d'un certain domaine multiconnexe de points. En d'autres termes, il s'agit de caractériser le groupe de difféomorphismes de l'anneau ou, dans le cas le plus général, du tore ${ }^{9}$. Il s'avère que la solution de ce problème de géométrie (qualitative) est essentielle pour la compréhension des solutions périodiques ou quasi périodiques dans le problème des trois corps et, d'une manière plus générale, pour connaître les conditions de stabilité d'un svstème dynamique global quelconque (comme le système solaire). Ce qui précède a en fait une signification philosophique profonde, à savoir que l'étude du comportement d'un sýstème dynamique au voisinage de certains " objets " représentés par des points et leurs orbites dans un espace de phase (ce concept aussi a été introduit par Poincaré), se ramène à l'étude des propriétés de certains objets inveriants sous l'action d'un groupe de transformations : orbites périodiques. invariants toriques, variétés invariantes stables et instables. Ces objets géométriques jouent un rôle fondamental dans l'organisation de la dynamique d'un système, et ils permettent d'en interpréter le comportement.

Les trois principaux caractères que nous avons attribués aux objets mathématiques se trouvent à être profondément liés dans le concept de symétrie. Ce concept joue un rôle de plus en plus fondamental dans toutes les branches des mathématiques et de la physique. qüil s’agisse de la physique moléculaire, de la cristallographie. de la biophysique, et plus récemment, des théories de jauge. Les symétries peuvent être soit de nature purement géométrique, soil de nature $d$ )namique, ou bien les deux à la fois. Les symétries dynamiques se laissent à leur tour distinguer en symétries globales et locales. Les symétries locales ont très peu de choses en commun avec les symétries globales. En effet, pour les svstèmes physiques qui admettent un groupe de symétries locales (c’est-à-dire un groupe invariant de transformations de jauge du type $\left.x(t)_{h} \equiv\left(x_{1}(t) h_{1}, \ldots, x n(t) h_{n}\right)\right)$, on ne peut concevoir aucune expérience qui permette de distinguer entre les deux orbites $x(t)$ et $x(t)_{h}$. Ainsi, $x(t)$ et $x(t)_{h}$ correspondent effectivement à la même orbite physique de $S$ (le système physique), exprimée dans des coordonnées intrinsèques différentes. L'idée de symétrie locale a conduit au développement des théories de jauge en physique, et elle est en partie à l'origine de la théorie des fibrés en mathématiques. Le tournant de la physique théorique actuelle consiste à éliminer les symétries globales des lois

9. Voir H. Poincaré, « Sur un théorème de géométrie ", Rend. Circ Mat Palermo, $33,1912,375$ 407. Pour un exposé éclairant sur les développements récents du sujet, voir $A$. Chanciner. C. Simó, "Mécanique céleste. Cent ans après la publication des "Méthodes Nouvelles " de Henri Poincaré *. Société Mathématique de France, journée annuelle 1996. 
physiques, et à trouver en même temps des lois dynamiques qui admettent un groupe (non abélien) de symétries locales ${ }^{10}$. Cependant, les symétries spatiales (groupe covariant de Poincaré) demeurent toujours des symétries globales, à moins d'inclure la gravité. De telles lois dynamiques sont exprimées sous la forme de théories de jauge. Cette situation pose un problème théorique très intéressant, quant à la manière de dériver des symétries internes globales, valables pour des modèles phénoménologiques ou des situations macroscopiques, à partir de symétries asymptotiques dynamiquement engendrées.

Dans le même ordre d'idées, un exemple très important est celui de la connexion que l'on suppose exister entre les théories (totalement) covariantes (comme la relativité générale), la thermodynamique et la théorie quantique des champs, connexion que demièrement l'on cherche à expliquer au moyen d'une propriété structurale clé des algèbres de von Neumann. Rappelons d'abord que la description physique des systèmes qui ne sont pas en général covariants, est fondée sur trois notions physiques élémentaires : les observables, les états et le paramètre temps. Les observables et les états déterminent la cinématique du système, le paramètre temps (ou le sousgroupe à un paramètre du groupe de Poincarć) permet d'en décrire sa dynamique. En mécanique quantique, de même que dans la mécanique classique, on a deux manières équivalentes de décrire le flot temporel : soit comme un flot dans l'espace des états (c'est l'approche de Schrödinger), soit comme un groupe d'automorphismes à un paramètre de l'algèbre des observables (c'est l'approche de Heisenberg). Dans la mécanique hamiltonienne classique, par exemple, les états sont représentés comme des points $s$ dans l'espace de phase $\Gamma$, et les observables comme des éléments $f$ de l'algèbre $A=C^{\infty}(\Gamma)$ des fonctions lisses dans $\Gamma$. L'hamiltonien $H$ définit un flot $\alpha_{t}^{s}: \Gamma \rightarrow \Gamma$, pour chaque $t$ (réel), dans l'espace de phase, et, par conséquent, un groupe d'automorphismes à un paramètre $\left.(\alpha f)=f\left(\alpha_{t}^{s} s\right)\right)$ dc l'algèbre des observables $A$. Cette façon de représenter les choses n'est plus valable dans les théories totalement covariantes, ni dans les théories relativistes qui incorporent le champ gravitationnel, et cela du fait qu'il est impossible d'y définir un flot temporel privilégié. Mais également parce que les lois dynamiques de ces théories ne peuvent pas être formulées en termes d'une évolution selon un seul paramètre temporel externe. Plus précisément, cette notion de flot temporel dépend de l'état du système. Qui plus est, une telle notion faible du temps n'a plus lieu d'être lorsqu'on inclut dans le modèle physique soit la thermodynamique, soit la mécanique quantique. La raison en est qu'en présence d'une " superposition "thermique ou quantique des géométries, la structure causale de l'espace-temps n'est pas conservée.

10. Pour une discussion des idées fondamentales sur ce sujet, voir J. Fröhlich, Non-Pernurbative Quantum Field Theory, Advances series in Mathematical Series, Vol. 15. World Scientific Publishing Co., Singapore, 1992. 
D'où l'apparition de grandes difficultés conceptuelles dans les théories quantiques de la gravité. Une idée intéressante, développée récemment ${ }^{11}$, consiste à essayer d'élargir la notion de flot temporel aux théories à covariance généralisée, mais en la faisant dépendre de l'état thermique du système. Plus précisément, la notion de flot temporel peut être étendue tout naturellement aux théories covariantes pourvu que : (i) on interprète le flot temporel en termes d'un groupe d'automorphismes à un paramètre de l'algèbre des observables; (ii) on attribue les propriétés temporelles du flot à des causes thermodynamiques; (iii) on premne sérieusement en considération I'idée que dans un système covariant général la notion du temps n'est pas indépendante de l'état, comme en physique non relativiste, mais qu'elle dépend plutôt de l'état dans lequel se trouve le système. Or, le fait qu'un état léfinit une famille d'automorphismes à un paramètre, c'est là une propriété fondamentale de l'algèbre de von Neumann. La relation entre un état $\omega$ aurlessus d'une algèbre et une famille d'automorphismes à un paramètre $\alpha_{t}$ appartenant à l'algèbre, est la source de la classification de l'algèbre de von Neumann, et donc au cceur de la théorie algébrique elle-même. Le lien entre un état thermique et un flot temporel peut être alors vu comme un cas particulier d'une telle relation générale. Cette observation conduit à des applications très larges de l'idée mentionnée plus haut et elle permet, en particulier, de relier un résultat mathématique profond à certaines propriétés physiques qui caractérisent les états dans la théorie quantique des champs. Les observables d'un système quantique forment une $C^{*}$-algèbre. Les états sont des fonctionnels linéaires positifs définis sur l'algèbre. Dans une théorie partiellement covariante, la définition de la théorie est complétée par I'hamiltonien, ou, ce qui revient au même, par une représentation du groupe (le Poincaré. Par ailleurs, dans une théorie à covariance généralisée on a seulement l'algèbre des observables de jauge invariants et les états. Étant donné un état, il existe un théorème (de Tomita-Takesaki) qui permet de construire un groupe d'automorphismes à un paramètre $\alpha_{f}$ de la clôture faible de l'algèbre, appelé le groupe modulaire. De cette manière, on peut élargir le postulat sur le temps thermique à la théorie quantique, en admettant que le temps physique soit le flot modulaire de l'état thermique. On obtient ainsi une définition du flot temporel physique dépendant de l'état daus le contexte de la théorie quantique covariante des champs. Une conséquence remarquable découle de l'hypothèse précédente. En effet, un résultat clé de la théorie algébrique de von Neumann est le théorème suivant: le flot modulaire, pourvu d'un automorphisme propre, est une propriété intrinsèque de l'algèbre, indépendante des états. En ce sens, l'algèbre de von Neumann est intrinsèquement un objet "dynamique ". Ainsi, si

11. Voir A. Connes and C. Rovelli, "Von Neumann Algebra Automorphisms and TimeThermodynamics Relation in General Covariant Quantum Theories *. Preprint IHES, Buressur. Yvette, $n^{\circ}$ 36, juin 1994. 
l'on interprète le flot modulaire en termes de flot temporel physique, ce résultat acquiert une signification physique profonde, à savoir que c'est la structure algébrique intrinsèque de l'algèbre des observables qui détermine le "flot temporel " compatible avec la théorie physique.

C'est bien pour les raisons que nous venons de voir, qu'il nous est difficile de partager (ou de ne partager qu'en partie) la position d'Alain Michel et, d'après lui, de Desanti et de Granger, quant au statut et à la signification des objets (idéalités) mathématiques. Ceux-ci seraient a des sortes d'objets qui ne sont accessibles que dans le système des possibilités réglées ouvertes par les relations qui les définissent. Une idéalité mathématique n'est en ce sens rien d'autre qu'une indication de procédure opératoire ou démonstrative. Sans doute, seule une procédure d'écriture symbolique (et le plus souvent purement idéale) fixe ces sortes d'objets, par des systèmes infinis d'architectures de signes, pour lesquels on peut définir des codes explicites de désignation. Mais les objets n'existent pas en dehors des systèmes de relations où s'inscrivent leurs propriétés: leur mode d'existence est en ce sens entièrement intra-théorique, relatif aux propriétés du système dans lequel ils sont accessibles". A. Michel précise que "dans l'expression de " contenu formel " [utilisée par Granger pour caractériser les objets mathématiques], " formel " est là pour souligner la nature fondamentalement symbolique du concept, comme de la comnaissance, mathématique, et même de toute connaissance d'objet en général ". Mais c'est là précisément la question. (À ce propos, le fait n'est pas d'avoir ou non un penchant pour les disciplines formelles - toute personne raisonnable ne niera pas qu'une des tâches importantes du travail scientifique consiste à donner une bonne axiomatisation pour tout domaine de phénomènes étudiés ${ }^{2}$, mais ce n'est pas son unique objectif ni d'ailleurs le plus fondamental.) Par ailleurs, nous n'ignorons évidemment pas l'importance des contenus formels en mathématiques (on vient de le voir à propos de la structure algébrique d'une théorie physico-mathématique, et nous allons le voir dans le cas du concept de groupe). Nous n'avons non plus aucune difficulté à admettre (et c'est là une thèse que nous avons soutenue dans l'ouvrage avec des arguments, croyons-nous, convaincants), d'une part, que les concepts mathématiques ne peuvent pas être obtenus à partir de matériaux sensibles, et d'autre part, qu'ils ne sont pas l'expression d'essences

12 Mais là encore, le fait de vouloir axiomatiser une théorie sert d'abord et avant tout à en mettre en évidence la structure mathématique fondamentale. Un exemple tout à fait éclairant et très important de nos jours est celui des théories topologiques des champs quantiques. Les axiomes communs à ces théories, de nature purement mathématique et apparemment indépendants de toute interprétation physique, se révèlent être en fait ceux auxquels satisfont plusieurs théories physiques. Qui plus est, chaque concept mathématique fondamental qui intervient dans ces axiomes est susceptible en principe d'une interprétation physique intéressante. En particulier le concept de cobordisme (dû à Thom) joue un rôle clé dans ces théories. Pour une présentation de ces théories, voir M. Atiyah, "Topological Quantum Field Theory •, Publications Mathématiques, Institut des Hautes Études Scientifiques (numéro dédié à René Thom), nº 68 (1988), p. 175-186. 
idéales séparées du processus de constitution des "objets" mathématiques eux-mêmes. Tout ce que nous visons à montrer est (i) premièrement, que les contenus formels ne s'identifient pas à l'ensemble des symboles et des règles qui les fissent, car ils recouvrent un domaine de sens beaucoup plus large ; (ii) deuxièmement, que les contenus formels d'un concept (ou d'une théorie) mathématique sont loin d'en épuiser leur signification. Il serait difficile d'affirmer, par exemple, que la nature du concept de connexion (concept spatial par excellence, qui possède tout à la fois une signification géométrique, algébrique et topologique), ou du concept du continu, soit seulement (ou surtout) symbolique. En vue de justifier une telle affirmation, faisons quelques brèves considérations sur ces deux concepts.

À partir des années 1970, on reconnaît que, d'un point de vue mathématique, la théorie de jauge est essentiellement une branche de la géométrie différentielle, précisément celle qui fait intervenir le concept d' "espaces fibrés " munis d'une "connexion " ${ }^{13}$. Cette notion joue un rôle absolument central dans la compréhension des rapports entre structures mathématiques et théories physiques et elle relie directement la géométrie à la physique, à tel point que l'on peut affirmer que les deux sont coextensives. Le concept géométrique de connexion est devenu ainsi un élément essentiel de la physique.

Ces développements montrent en particulier qu'à chaque entité physique correspond un concept de la géométrie et de l'analyse différentielle globales. Par exemple, l'intensité du champ s'identifie avec la courbure de la connexion ; l'intégrale d'action “ n'est rien d'autre "qu'une mesure globale de la courbure. Certains invariants topologiques et algébriques appartenant à la théorie des classes caractéristiques se sont révélés très appropriés pour décrire la charge de la particule au sens de Yang-Mills. De façon plus générale, on peut faire correspondre directement les concepts de la théorie des champs de jauge à ceux de la géométrie et topologie différentielles des fibrés. Mais comment faut-il comprendre au juste la nature d'une telle correspondance? Inspirés par une idée déjà avancée par Hermann Weyl en des termes quelque peu différents ${ }^{14}$, nous soutenons la thèse selon laquelle au fond le physique n'est que le géométrique en acte. C'est dire que, non seulement la géométrie est productrice de structures idéales, d'universaux sémantiques comme la variété, le groupe, la courbure, la connexion, le fibré, mais encore elle participe des propriétés des entités physiques et des qualités des phénomènes. On pourrait en fait aller jusqu'à postuler qu'il doit y avoir en principe, selon la théorie et la classe des phénomènes envisagées, une

13. Voir J.-P. Bourguignon et H. B. Lawson, " Yang-Mills Theory: Its Physical Origins and Differential Geometric Aspects ". Annals of Mathematics Studies: Seminar on Differential Geometry, S.-T. Yau (Ed.), Princeton University Press, 1982, p. 395-421. Pour un exposé complet sur le sujet, voir A. S. Schwartz, Quantum Field Theory and Topologr; Springer-Verlag, Berlin, 1993.

14. Voir H. Weyl, "Reine infinitesimale Geometrie *, Mathematische Zeitschrift, 2 (1918), p. 384 411. 
structure géométrique continue ou discrète sous-jacente à toute phénoménologie physique donnée. Pour s'en convaincre, il suffirait de rappeler que certains grands principes de symétrie géométriques (ou certains groupes, ce qui revient au même) se convertissent en des principes dynamiques qui, eux, sont responsables de bon nombre de changements d'état affectant les phénomènes physiques. Ne faudrait-il pas alors affirmer qu' " à l'origine était la symétrie ou le groupe..." ? Cependant, ce concept n'est pas uniquement de l'ordre de l'abstrait et les propriétés mathématiques qu'il contribue à mettre en évidence ont à la fois une puissance explicative et une force productrice d'un monde fait de forces, d'interactions et d'énergie... L'intelligibilité mathématique de ce monde n'est pas ainsi une chose séparée de l'intelligibilité du réel lui-même ${ }^{\mathbf{5}}$.

Passons maintenant au problème du continu. La question de savoir comment arriver à donner une représentation non ensembliste du continu présente un intérêt indéniable ${ }^{16}$, mais elle n'a pas jusqu'à maintenant trouvé l'attention que pourtant elle mérite. Il faudrait, pour cela, parvenir à une caractérisation purement géométrique (topologique) du continu, qui ferait notamment intervenir l'homogénéité et la connexité de l'espace, encore que cela serait une condition nécessaire mais non pas suffisante. Mais il est bien connu que d'un point de vue mathématique, le modèle général et communément accepté pour penser le continu a été le modèle ensembliste de la droite réelle $R$ proposé par Cantor-Dedekind. Le continu y serait engendré à partir du discret (de la suite des entiers naturels) - donc à partir de la générativité de l'arithmétique - par complétion des coupures définies sur les rationnels. Cette idée d'une priorité logique du discret s'appuie sur le fait apparemment indiscutable que la suite des entiers naturels est. typiquement une suite discrète d'entités, le nombre ; chacun est un point qui est séparé de tous les autres nombres points.

À l'encontre de la conception, qui veut que le continu soit le produit (fini en acte) d'une générativité purement arithmétique (formelle par excellence donc), on pourrait opposer le continu archétype que l'on peut imaginer comme un espace ayant la propriété d'une homogénéité qualitative parfaite, au sens que deux " points "y sont toujours équivalents par un glissement continu (éventuellement seulement local) de l'espace sur lui-même. Cependant, pour qu'une telle image ne paraisse pas contradictoire, il faut arriver à faire l'économie de la notion de " point ", qui présuppose déjà une brisure de l'homogénéité spatiale ce qui, d'un point de vue mathématique, apparaît une tâche plutôt difficile. Il faudrait peut-être, pour réussir à surmonter une telle difficulté, faire appel à des notions purement qualitatives

15. Il revient surtout à René Thom d'avoir montré que ce postulat philosophique est susceptible d'être développé dans un programme mathématique et phénoménologique très fécond et précis. Voir, entre autres, Esquisse d'une Sémiophysique, InterÉditions, Paris, 1988, et Apologie du logos, Hachette, Paris, 1990.

16. Voir H. Weyl, Das Kontinuum, Veit, Leipzig, 1918 
de topologie, telle que celle de déformation continue d'une surface dans une autre (plongement, immersion, etc.) ${ }^{17}$.

Il est une conception très répandue selon laquelle le continu s'obtient en intercalant indéfiniment un nombre entre deux autres nombres qui ne sont pas égaux. C'est l'idée, entre autres, de Poincaré. Ainsi, toute nouvelle suite d'éléments formerait encore un ensemble dénombrable : un ensemble dense, comme les rationnels, suffit à réaliser la construction de Poincaré. Les coupures de Dedekind ne définissent qu'un ensemble au plus infini dénombrable de nombres réels. Le continu mathématique, doué de la propriété d'être un ensemble parfait (identique à son dérivé : tous ses points sont points-limites ou d'accumulation), connexe, non dénombrable, est plus riche que les ensembles qu'on atteint par les procédés de Dedekind et de Poincaré. Du point de vue de la théorie des ensembles, le continu ne s'engendre pas par répétition du point, comme le croyait Poincaré. La connexité ne suffit pas à définir le continu. Un ensemble peut être parfait et totalement discontinu, ainsi l'ensemble triadique $K$ de Cantor. Cet ensemble est construit en sorte que les intervalles qui le composent sont disjoints. Il n'est nulle part dense : tout segment de l'intervalle fermé $[0,1]$ renferme des points du complémentaire de $K$. L'ensemble $K$ est au moins dénombrable ; il a la puissance du continu.

Selon une autre conception, qui remonte à Aristote et qui est encore présente chez des mathématiciens comme Riemann, Clifford, Peirce, Veronese, Husserl et Weyl, le continu n'est pas à proprement parler composé de points consécutifs ou en contact. Divisible en intervalles eux-mêmes divisibles, le partage de ces intervalles se poursuivant à l'infini, un continu est divisible en puissance. Enlever un point d'un continu unidimensionnel le décompose en deux ouverts disjoints : cela détruit la continuité. Donc, un continu n'est pas formé de points en acte. Un continu qui est en puissance ne contient rien d'indivisible ; il faudrait pour cela admettre des figures du continu qui ne sont pas ponctuellement divisibles, comme des points qui, au lieu de se diviser, se contractent ou se dilatent en ne changeant pas de forme (la topologie différentielle nous en offre des exemples, et la biologie nous montre des processus morphogénétiques qui rappellent un tel modèle), ou des lignes insécables, ou des cordes qui se nouent et se dénouent suivant une application continue (deux nouds peuvent être isotopes, ou encore des surfaces qui, après avoir subi certaines transformations, ont la propriété de revenir à leur forme originelle). Des glissements et des rotations sont aussi des modèles de continuité. Alors que retirer un nombre d'un continu linéaire divise ce continu en supprimant sa connexité ; nommer ou choisir un point actualise ce point: dans le continu le point est donc tout de même en

17. Pour une tentative qui va dans ce sens, voir L. S. Husch, "Imbedding of continua ", dans Geometric Topology and Shape Theory, S. Mardésic \& J. Segal (Eds). Lectures Notes in Mathematics 1283, Springer-Verlag, Berlin, 1987, p. 125-131. 
puissance. Le continu est encore moins accessible par composition ; s'il l'était, il serait composé d'une infinité actuelle d'éléments. Aristote rejetait les continus ouverts et n'admettait que les continus bornés. René Thom a repris cette idée et l'a développée dans une direction tout à fait stimulante pour la pensée. Ces ouverts bornés seraient des continus compacts (des unités en acte) déterminés par la surface qui les borde à distance finie. Divisible à l'infini (en puissance), le continu n'est pas composé. S'il l'était, il constituerait un infini actuel ; le recomposer à partir de nombres ou points que nous y actualisons en les distinguant, ne donnerait qu'un infini potentiel. Le fait d'admettre un continu divisible à I'infini permet d'éviter le paradoxe de la composition : comment une étendue se formerait-elle de points inétendus?

Il n'est pas ainsi complètement déraisonnable d'affirmer que le continu linéaire n'est peut-être pas le seul modèle de continu mathématique ; en tous cas il n'est pas le seul modèle de continu réel possible ${ }^{18}$. Déjà l'idée d'un continu ayant une structure topologique non discrète permet une nouvelle définition de la connexité et de l'homéomorphisme qui fait davantage appel à la nature de l'espace plutôt qu'à celle des nombres (des points). La théorie topologique des surfaces et la théorie des nouds, dont elle est issue, peuvent nous aider à mieux comprendre. La topologie d'un noud n'est pas une propriété de la courbe qui le représente, mais de l'espace qui le contient. Ainsi, les manières de se déplacer dans l'espace $S$ (le complément du nœud) sans rencontrer le nœud qui y est plongé caractérisent ce nœud. Considérons le cas très simple du nœud de trèfle. On peut représenter plusieurs chemins fermés et orientés, partant d'une origine arbitraire $P$, parcourant l'espace qui entoure ce noud et revenant au point $P$. Une infinité de chemins partent de $P$, et certains d'entre eux ont la même topologie; deux chemins sont équivalents s'il existe une manière de déformer l'un en l'autre. Cette déformation, appelée homotopie, consiste à étirer, contracter, voire croiser un chemin, en maintenant ses extrémités au point $P$ et sans jamais couper le noeud.

Le continu mathématique, tel que défini plus haut, ne peut pas être appliqué pour définir le continu physique, sauf pour la propriété de la divisibilité à l'infini qu'ils partagent; mais ils diffèrent quant à d'autres propriétés importantes. On ne peut pas assimiler un champ de matière ni un morceau d'espace physique au continu mathématique. Ce qui leur est propre n'est pas d'admettre une infinité de points, ni le fait que la matière soit nécessairement divisible à l'infini. Un champ physique est essentiellement de nature continue. Les interactions entre les particules ont lieu grâce à l'existence d'un milieu qui propage ces interactions : les champs de jauge qui correspondent aux symétries des particules. En fait, une interaction

18. Pour une réflexion intéressante à ce sujet, voir G. Longo, * Le continu mathématique, de l'intuition à la logique , Preprint de l'École Normale Supérieure, Paris, décembre 1996. 
correspond à un groupe (au sens mathématique) de symétries. Plus précisément, les interactions entre les particules d'un champ (par exemple, électromagnétique) possèdent une structure de groupe continu, ou de Lie: elles se transforment donc selon les opérations de ce groupe, qui dépendent d'un ou de plusieurs paramètres variant continûment. On voit par là que la structure géométrique d'un espace physique (même s'il est sans matière) devient le support des lois de conservation des entités physiques (des forces, des charges, des impulsions, etc.).

Un autre point essentiel qu'il faut saisir est que l'espace n'est pas simplement un ensemble de points quelconques, mais un ensemble d'objets possédant un système défini de propriétés, qui constitue la structure de l'espace. Comme Weyl et Brouwer l'ont bien montré, le concept de variété ne peut pas être réduit à celui d'ensemble. Pour Weyl, la caractéristique essentielle d'une variété est d'être un espace continu, avant d'être un continu linéaire : une variété n'est pas un ensemble qui se laisse décomposer en des éléments discrets ; autrement dit, ce n'est pas sur la relation de l'élément à l'ensemble, mais plutôt sur celle de la partie au tout que l'analyse du continu doit être fondée. Dans son grand ouvrage Die Idee der Riemannschen Fläche (1913), Weyl a très bien mis en évidence que toutes les propriétés qui caractérisent une surface de Riemann, ou toute variété qui lui est isomorphe, peuvent être ramenées uniquement à celles de continuité (Kontinuität) et de voisinage (Umgebung). En effet, une variété est continue lorsque ses points " adhèrent " entre eux, autrement dit, lorsqu'il devient possible d'y définir une carte de voisinages locaux. On dira qu'un espace topologique est connexe s'il n'est pas la réunion de deux domaines disjoints, ou lorsque deux points quelconques peuvent être joints par une courbe continue, entièrement située à l'intérieur d'un domaine de la variété (connexité par arcs). C'est une vision essentiellement structurale et qualitative de l'espace qui se dégage des travaux de ces mathématiciens.

On touche là à l'un des problèmes majeurs de toute théorie de I'intelligibilité, à savoir comment expliquer le passage du virtuel à l'actuel. Il appartient, nous semble-t-il, à une recherche phénoménologique à vocation essentiellement géométrique d'expliquer les principes et les lois qui soustendent une telle relation constitutive. $\grave{A}$ ce propos, une grande idée dont Riemamn a jeté les bases et au développement de laquelle Weyl a contribué de façon décisive, est que tout ce qui dans l'univers a une existence réelle (au sens de la physique) n'est qu'une manifestation de sa structure géométrique. Mais les phénomènes eux-mêmes et leurs modes d'apparaître sont restés en dehors de cette recherche. Si l'on exclut quelques réflexions philosophiques éparses - par ailleurs remarquables - de Riemann sur les rapports entre matière et esprit, et certaines analyses beaucoup plus fouillées faites par Weyl dans quelques-uns de ses ouvrages de nature philosophique, la structure du monde phénoménal n'a pas vraiment fait l'objet d'études approfondies avant que certains Naturphilosophen, Husserl et les théoriciens de la Gestalt, ne lui 
consacrent leurs travaux les plus remarquables. Leurs idées, cependant, bien que d'un grand intérêt, apparaissent insuffisantes, surtout en ce qui concerme la compréhension mathématique de ce monde phénoménal qu'ils ont délaissée pour une description principalement phénoménologique. René Thom a fait une contribution essentielle à l'éclaircissement et peut-être à la solution d'un tel problème, grâce notamment à sa théorie des catastrophes.

Bref, si dans les théories géométriques les plus abstraites (théorie des groupes finis et géométrie algébrique), la création de nouveaux objets mathématiques est en général rendue possible par une sorte de générativité formelle interne à la théorie, il n'en va pas de même dans beaucoup d'autres branches fondamentales de la géométrie, plus directement liées à la physique théorique, où il s'agit plutôt de comprendre le rôle joué par certains grands principes et où l'engendrement (de nature géométrique) de formes réelles est de loin plus significative qu'une générativité purement formelle. De ce point de vue, il n'est pas très surprenant que depuis quelques années la théorie topologique des nœuds, par exemple, soit devenue un outil conceptuel essentiel pour comprendre certains phénomènes électromagnétiques dans la théorie de jauge ou des phénomènes quantiques" ${ }^{19}$, ou que la notion de singularité soit devenue tout aussi importante pour essayer d'expliquer des questions liées à l'origine de l'univers et à la forme topologique de l'espace. Ce qui précède nous conduit à avancer une hypothèse très générale :

Hypothèse. Il y a de grands principes en physique, qui montrent que les changements d'état physique de la matière sont à reconduire à un principe de permanence de certaines formes topologiques et au fait que, dans le passage d'un état à l'autre de la matière, la structure géométrique de ces formes puisse varier et se modifier sans qu'elle en soit pour autant détruite.

Pour les mêmes raisons que nous venons de rappeler, il nous est également difficile d'accepter l'interprétation que Jean Petitot donne dans son article du conventionnalisme géométrique de Poincaré, qu'il qualifie de transcendantal. Des objections peuvent aussi être formulées à l'égard de la conclusion qu'il tire d'une telle interprétation. En s'appuyant sur des thèses bien connues de Poincaré, à savoir : i) qu'une géométrie n'est ni vraie ni fausse, c'est-à-dire qu'elle ne possède pas de vérité expérimentale, car ses propositions ne portent pas sur l'espace mais uniquement sur les corps qui existent dans l'espace ; ii) que le concept de groupe est une forme a prioride l'entendement, J. Petitot énonce des thèses d'une portée philosophique plus générale. Résumons-les : 1) “ la géométrie est une convention, c'est-à-dire un a priori (grammatical si on veut) de l'expérience. Elle fixe un langage de description et ne possède pas de vérité expérimentale. [...] C'est un a priori au sens des conditions de possibilité déterminantes"; 2) “l'a priori de la géométrie se ramène essentiellement à l'a priori des groupes... n; 3) l'a priori

19. Voir T. Kohno (ed.), New Developments in the Theory of Knots, World Scientific Publishing Co., Advanced Series in Mathematical Physics, Vol. 11, Singapore, 1989. 
est une affaire de choix, c'est-à-dire qu'il obéit à un critère pragmatique, plus précisément à celui de la commodité.

Selon Petitot, ces thèses reviennent dans le fond à affirmer que a l'objectivité physique dépend des conventions fixant la qualification géométrique des données empiriques, en particulier le fait que les symétries des théories sont mathématiquement déterminantes pour le contenu physique de ces théories... ". On notera que l'interprétation proposée par Petitot du conventionnalisme géométrique de Poincaré présente curieusement certaines affinités avec l'interprétation proposée par certains philosophes néo-empiristes comme H. Reichenbach ou A. Grünbaum. En effet, les deux ont mis l'accent sur le caractère linguistique et pragmatique des conventions géométriques. Il y a d'ailleurs, nous semble-t-il, une certaine confusion à ce sujet. Elle consiste à présenter comme complémentaires le fait que les a priori géométriques soient autant de conditions de possibilités des phénomènes (il s'agit là d'une reprise de l'idée kantienne des concepts mathématiques comme des conditions a priori de possibilité objective des phénomènes), et le fait que ces mêmes a priori soient de simples conventions linguistiques. D'abord, cela n'explique pas comment il se fait que certains concepts géométriques, qui sont effectivement des formes a priori, se trouvent à être constitutifs d'un domaine d'objets mathématiques. Ensuite, le concept de groupe n'est pas seulement un a priori de notre entendement, car il est en même temps un concept clé de toute théorie de la nature qui se veut intelligible ; il explique le “ réel ». Enfin, le groupe n'est pas une simple convention, car de lui dépend le fait que tout objet mathématique puisse se constituer selon différents niveaux de structures - éventuellement isomorphes (à chacun de ces niveaux correspond ainsi un mode d'existence -- virtuel - de l'objet abstrait groupe). Mais de lui dépend également la constitution des propriétés essentielles des entités physiques ainsi que leur plus ou moins grande stabilité ; par exemple, un changement dans la configuration du groupe principal d'un certain espace peut être responsable du changement de phase en physique.

Nous soutenons ici la thèse que non seulement la géométrie est productrice d'universaux sémantiques comme la courbure, la variété, le groupe, la connexion, etc., mais qu'elle est également, en un certain sens, ontologiquement enracinée dans le réel. De plus, elle est impliquée dans son devenir, car elle participe des propriétés des entités physiques (notamment par l'action constitutive de ses principes de symétrie) et des qualités des phénomènes ; il suffit, à ce propos, de penser au rapport entre les propriétés géométriques de l'espace perceptif et les qualités sensibles. Enfin, il faut bien voir que le monde des formes à la fois perceptives, inorganiques et organiques, leur constitution et évolution, dépendent intimement de certaines lois de transformations géométriques fondamentales et de facteurs dynamiques qu'elles sont à même d'engendrer. Ainsi, par la thèse que nous avons soutenue plus haut, à savoir que le physique n'est que le géométrique en 
acte, il faut entendre les deux choses suivantes : (i) que l'espace physique est une déformation de l'espace euclidien, mais que d'autres déformations seraient possibles si l'on envisageait un espace différent, de plus grande dimension et avec une structure plus riche; (ii) que le monde des formes concrètes que l'on observe ne réalise en fait qu'une partie des formes géométriques abstraites. Celles-ci expriment donc des potentialités qui n'arrivent pas à terme, ce qui peut être dû soit à des contraintes globales, soit à des accidents locaux et/ou contingents.

De tout ceci il résulte, entre autres, qu'on ne peut expliquer les propriétés physiques des corps indépendamment des propriétés géométriques qui les caractérisent intrinsèquement ; pour comprendre les processus qui donnent lieu à ces mêmes propriétés, il faut ainsi d'abord comprendre les lois de transformation des objets mathématiques qui se laissent définir sur un certain type d'espace substrat. Par exemple, pour connaitre les propriétés physiques d'équilibre d'une classe de corps, il suffit de déterminer les figures d'équilibre géométriques d'une certaine surface ellipsoïdale. Ou encore, pour savoir s'il existe des solutions périodiques dans le problème des trois corps (en mécanique céleste), il faut pouvoir démontrer un théorème de géométrie (le théorème de Poincaré-Birkhoff) ${ }^{20}$.

Reprenons maintenant un certain nombre de points épistémologiquement importants abordés dans le livre, que nous aimerions développer ici.

\section{A propos de l'antériorité logique et intuitive de la géométrie d'Euclide}

Une des critiques que Husserl a adressées notamment aux conceptions de Riemann s'appuie sur un argument de nature plus philosophique que mathématique, au sens technique et général du terme : la théorie de la courbure ne peut servir à élucider les fondements logiques de la géométrie. Selon Husserl, la vraie théorie géométrique demeure l'euclidienne, et la valeur des nouvelles conceptions mathématiques (de Riemann et d'autres) tient uniquement à leur pouvoir de généralisation logique de la géométrie d'Euclide. C'est le fait de penser que les variétés euclidiennes ne sont qu'un cas d'espèce parmi une infinité d'autres cas possibles de variétés qui, aux yeux de Husserl, infirme la théorie de Riemann et d'autres mathématiciens. Il affirme que l'espace euclidien doit être présupposé dans toute tentative de généralisation ou de construction d'autres espaces. On ne peut pas définir une surface ou une variété de dimension $>2$ en faisant abstraction de l'espace euclidien à trois ou à $n$ dimensions, car, en fait, l'espace euclidiencartésien $R^{N}$ représenterait le cadre “ naturel " (et “ absolu ») de la théorie des surfaces et variétés courbes. C'est dire que la géométrie euclidienne jouirait

20. Voir à ce propos L. Boi, "Mathématiques qualitatives, théorie des groupes et conception de l'espace chez Poincaré *, Preprint de l'Institut des Hautes Études Scientifiques, Bures-surYvette, janvier 1996, p. 1-24. 
d'un statut spécial face aux autres théories géométriques, dans la mesure où ses concepts seraient de l'ordre de la « nécessité logique "plutôt que de celui de la construction mathématique. Husserl croit, dans un certain sens, à une sorte de primauté de la géométrie euclidienne sur les nouvelles théories géométriques.

Mais le fait de considérer l'espace euclidien comme primordial a en luimême une signification mathématique profonde, ce qui d'ailleurs confêre à l'argumentation de Husserl une valeur et une importance générales indéniables. L'espace euclidien est essentiel, non seulement parce qu'il représente la réalité physique, mais encore et davantage parce qu'il est un " centre organisateur "; il est l'espace le plus dégénéré, celui qui a le plus grand groupe d'automorphismes. On peut, par exemple, penser que tous les espaces riemanniens à deux dimensions se produisent comme des sous. espaces d'un espace euclidien à trois dimensions, ou que des volumes non cuclidiens tridimensionnels admettent un plongement dans un espace euclidien de dimension $\geq 4^{21}$. Par ailleurs, on peut, dans un certain sens, concevoir l'espace physique comme une déformation de l'espace euclidien. C'est la géométrie de cet espace qui se reprojette dans l'espace euclidien comme "Physique ". La compréhension d'un objet géométrique comme déformation d'un objet plus dégénéré, plus facile à étudier à cause de ses nombreux automorphismes, est un des paradigmes de la géométrie moderne.

D'après Husserl, la théorie philosophique de l'espace de Riemann apparaît non fondée du fait que son idée principale, selon laquelle on ne doit attribuer à la géométrie euclidienne qu'une signification contingente (eine ganz zufälige Bedeutung), est complètement erronée. C'est le contraire qui est vrai : la géométrie euclidienne garde malgré l'existence des nouvelles théories un statut privilégié. Loin d'être contingente, la géométrie euclidienne est en quelque sorte "inscrite" dans la nature de l'espace (inhérente à l'espace) ; elle lui appartient comme une "donnée originaire ", au point qu'elle en constitue une espèce de principe « universel ".

Quant au contenu philosophique de la pensée de Riemann, Husserl ne se borne pas à en critiquer telle ou telle idée, mais il le conteste radicalement. À son avis, le plus grand tort du mathématicien aurait été de réduire la signification de la géométrie euclidienne à une partie de la théorie mathématique générale des variétés et d'en faire ainsi un chapitre parmi d'autres de la géométrie différentielle. D'une manière générale, Husserl semble sous-estimer l'importance du fait que Riemann conçoit celle-ci d'une façon beaucoup plus large qu'elle ne l'avait été avant lui : outre l'étude des courbes et des surfaces dans l'espace tridimensionnel ordinaire, elle envisage désormais ces figures comme des "objets " mathématiques autonomes, et elle considère en même temps d'autres objets géométriques tels que celui de

21. Pour de magnifiques exemples de ce type, voir W. Thurston, Three-Dimensional Geometry and Topologr, Princeton University Press. Princeton, 1997. 
variété différentiable. Ce ne sont pas les lois analytiques abstraites de ces objets qui intéressent au premier chef Riemann, mais plutôt leurs structures mathématiques essentielles. Dans l'histoire des mathématiques modernes, Riemann a été celui qui fait passer la pensée géométrique des lois à celle des structures : des mathématiques, il a incontestablement une conception structurale.

Une telle conception comporte déjà un dépassement d'une vision purement logique ou formaliste des mathématiques. Elle ne peut pas nos plus être rapprochée de l'idéal bourbakiste des mathématiques comme de théories déjà " propres "et achevées, bien formalisées dans des structures algébriques figées au-delà desquelles il ne faut surtout pas chercher une signification autre. En revanche, Riemann voit les mathématiques en quelque sorte comme un organisme vivant doué d'un dynamisme interne, et dont les concepts et les structures qui lui appartiennent prennent naissance dans un mouvement même de la pensée qui fait constamment appel aux ressources de l'intuition et de l'imagination. Ces structures sont des entités idéales, des formes intelligibles habitant un univers abstrait, et en ce sens elles sont virtuelles, mais elles sont également des modèles s'actualisant dans les phénomènes réels. Elles s'inscrivent dans ce mouvement vital de la pensée par lequel nous essayons d'idéaliser les phénomènes réels pour être plus à même d'en élaborer des représentations signifiantes. Ce mouvement, dont une partie fondamentale des mathématiques s'inspire constamment et tire ses plus belles créations, échappe au critère strict de vérité logique fou axiomatique) en lequel tant de mathématiciens et de philosophes ont prétendu figer la pensée mathématique et conformément auquel ils ont voulu en épuiser les contenus. Ainsi, la conception qu'a Riemann des mathématiques comporte, pour reprendre les mots d'A. Lautman, la solidarité du tout et de ses parties (du local et du global), la transformation des propriétés de relation en propriétés intrinsèques (sans quoi les relations entre les objets mathématiques n'auraient qu'une signification formelle et statique) et le passage des a êtres "imparfaits à des "êtres " pleinement définis. Ce sont là autant de moments d'organisation structurale qui confèrent aux être mathématiques une ascension vers l'achèvement par quoi on peut dire qu'ils existent. "Mais cette existence ne se manifeste pas seulement en ce que la structure de ces êtres imite les structures idéales auxquelles ils se laissent comparer : il se trouve que l'achèvement d'un être est en même temps genèse d'autres êtres, et ce sont là des relations logiques entre l'essence et l'existence où s'inscrit le schéma de créations nouvelles ${ }^{22}{ }^{n}$.

On peut retenir quelques points importants de la position de Husser ainsi que de sa critique à Riemann. Limitons-nous ici à considérer celui qui concerne ce que nous avons appelé la " réduction analytique " du problème de l'espace. L'idée est qu'on ne peut pas traiter l'espace (l'espace euclidien

22 Voir A. Lautman, Essai sur l'unité des mathématiques et divers écrits, Union générale d'édition, Paris, 1977 , p. 81 . 
des phénomènes) comme un simple exemple de variété à trois dimensions, posé par une définition purement analytique. Une telle simplification peut suffire tout au plus pour caractériser les relations formelles de l'espace dans le cadre d'une théorie mathématique des formes pures, mais elle ne peut en aucun cas prétendre épuiser la signification géométrique du terme espace, qui renvoie à d'autres contenus que le contenu formel. La théorie analytique (formelle) des variétés ne nous aide donc pas à expliquer la signification proprement géométrique de notre espace. Une telle explication peut uniquement résulter, selon Husserl, d'une connaissance des phénomènes de nature spatiale, qu'ils soient psychologiques, physiologiques, optiques ou physiques. Autrement dit, la compréhension des structures de l'espace et l'explication de la nature de ces phénomènes ne sont pas deux choses séparées.

En opposition à l'espace intuitif (Anschaungsraum), Husserl définit l'espace de la pensée scientifique (den Raum des wissenschaftlichen Denkens), c'est-à-dire l'espace géométrique, comme " une figure conceptuelle logiquement élaborée à partir de la représentation de l'espace appartenant à la conscience extra-scientifique (ausserwissenschaftlichen Bewusstseins); de l'espace géométrique on ne dira plus qu'il est représenté intuitivement ou qu'il est représentable, mais seulement qu'il est un objet de pensée ${ }^{23}$. L'analyse du concept géométrique d'espace doit consister en cette élaboration logique (logische Bearbeitung), c'est-à-dire en la qualification de la genèse de l'espace de l'intuition par l'espace de la géométrie pure "24. À la différence de l'espace intuitif, les représentations géométriques des formes spatiales ne gardent plus aucune trace de l'intuition, elles tirent leur origine de concepts formés logiquement. Husserl définit quelque part une telle élaboration logique comme " l'idéalisation de l'empirique, le procédé en devenir qui fait passer des intuitions extérieures aux vraies formes spatiales ». Plus tard, il qualifiera l'idéalisation géométrique de processus de pensée (Denkprozess), et il définira les concepts géométriques comme des concepts idéaux (voir Ideen I, § 74).

Il y a sans doute un fond de vérité dans le raisonnement par lequel Husserl met en évidence la signification profonde de l'espace euclidien, et dont la portée va d'ailleurs au-delà du rôle mathématique certes essentiel qu'il a joué pour l'élaboration de la géométrie et de la physique classique. Voici quelques-unes des raisons qui expliquent cette signification profonde. On mentionnera d'abord le fait que le groupe euclidien d'automorphismes de l'espace possède une structure très riche; ensuite, le fait que ses propriétés géométriques et l'ensemble des transformations auxquelles elles peuvent être soumises se laissent définir par l'action globale de son groupe

23. On voit ici que la position d'A. Michel et de G. Granger est au fond très proche de celle de Husserl.

24. Voir H. Husserl, Gesamnelte Werke, vol. 21 : Studien zur Arithmetik und Geometrie textes de 1886-1901), herausgegeben von I. Strohmeyer, Martinus Nijhoff, The Hague, 1983, p. 275-276. 
de symétries; enfin, le fait que tous les autres espaces qui sont concevables d'un point de vue mathématique peuvent être vus comme des ouverts de l'espace euclidien abstrait à $n$ dimensions - il ne faut pas oublier qu'une variété se définit par un atlas de cartes, et chaque carte est un ouvert euclidien. À ce qui précède, il faut ajouter le fait que l'espace euclidien est essentiel pour que les objets externes se constituent en tant qu'objets mentaux dans notre intuition. Une raison non moins importante de la nécessité conceptuelle de l'espace euclidien consiste en ce qu'il constitue le substrat naturel sur lequel reposent les fondements biologiques des organismes vivants, de leur développement et de leurs actions. Il faut enfin voir que la nature euclidienne de notre espace est une contrainte universelle à laquelle il est difficile d'échapper. Par exemple, elle est sous-jacente à l'existence de l'asymétrie gauche-droite de notre corps; le simple geste de nouer une ficelle pour former un nceud implique l'espace euclidien; elle intervient également dans la solution de petits problèmes de mécanique et de dynamique de tous les jours. Toutes ces raisons font d'ailleurs que la signification de l'espace euclidien ne peut pas être réduite à une liste d'axiomes purement formels.

Il n'en demeure pas moins qu'il serait une grave erreur épistémologique que de vouloir attribuer aux espaces abstraits non euclidiens une signification uniquement mathématique, et que de penser qu'ils n'ont pas eu au fond de conséquence pour la théorie de la connaissance. Or, c'est précisément leur construction qui est à l'origine d'une transformation profonde dans les conceptions philosophiques sur l'espace et le temps, la réalité physique et l'expérience. Un tel point de vue interdirait en particulier de comprendre les développements les plus importants dans les mathématiques et la physique théorique récentes. Ceux-ci peuvent être schématiquement caractérisés comme suit : (i) l'approfondissement de l'étude des propriétés topologiques des variétés différentiables; (ii) la floraison de nouveaux espaces abstraits de dimensions $n$ (avec $n$ quelconque - par exemple, les espaces de Hilbert), où le nombre de dimensions, loin d'avoir une fonction purement analytique, apparaît de plus en plus lié, d'une part, à la possibilité de comprendre certains problèmes de géométrie théoriquement très difficiles, et d'autre part, à la possibilité de donner une explication plus satisfaisante de l'univers physique à l'échelle quantique; (iii) la reconnaissance du rôle essentiel qu'ont les concepts géométriques dans la détermination mathématique de divers phénomènes naturels. 


\section{Intuition spatiale et theorie de l'espace chez Clifford}

L'originalité du travail mathématique de Clifford a été d'avoir montré les analogies importantes entre les conceptions de Grassmann et de Hamilton et la géométrie non euclidienne des espaces elliptiques. Son résultat fondamental a consisté en la construction d'une algèbre géométrique, c'est-àdire d'une théorie mathématique où les composantes algébrique et géométrique apparaissent comme deux aspects concurrents, bien que distincts, de la même méthode mathématique, deux moments progressifs de l'appréhension des objets mathématiques. L'algèbre n'est plus conçue comme un simple langage symbolique, un ensemble de techniques et d'opérations permettant de manipuler aisément et avec une économie de la pensée, les relations abstraites entre certains objets mathématiques. À ce propos, limitons-nous ici à mentionner deux points.

(i) La signification d'une théorie algébrique se trouve associée chez Clifford à un certain développement de la genèse d'idées mathématiques simples (ces idées ne sont pas données à l'avance, mais elles sont engendrées par la pensée) et aux transformations conceptuelles (virtuelles) dont ces idées sont susceptibles.

(ii) Il est possible de faire correspondre un contenu géométrique précis à chaque concept algébrique abstrait, ce qu'on peut appeler aussi une interprétation. Par ailleurs, les opérations algébriques n'existent pas sans produire de contenus géométriques, et ceux-ci, à leur tour, sont le plus souvent à l'origine des formalisations algébriques. Ce lien intime entre algèbre et géométrie ressort clairement dans la théorie des biquaternions, développée par Clifford et conçue comme une généralisation de la théorie des quaternions de Hamilton, mais également dans ses nouvelles idées sur la géométrie non euclidienne des espaces elliptiques. Dans ses recherches sur les propriétés géométriques qui caractérisent l'espace elliptique, Clifford montre que la théorie de l'espace est susceptible de plusieurs extensions fondamentales, pourvu qu'il soit possible d'enrichir de nouveaux " objets " mathématiques un domaine déjà existant, et/ou de donner plusieurs interprétations différentes, encore que compatibles, d'un même objet nathématique.

D'où d'ailleurs une conséquence philosophique importante : il apparaît difficile d'opposer, comme Kant le prétendait, l'intuition au concept, autrement dit, l'espace comme forme de la sensibilité externe à l'espace comme cadre d'une théorie axiomatique formelle. l'ensemble des concepts géométriques construits avant toute perception. Il est vrai cependant qu'il faut distinguer les deux types d'espaces pour être à même d'en apprécier leurs contenus aussi bien intuitifs que formels. Alors qu'on peut très bien concevoir des espaces abstraits sans qu'aucun contenu sensible ne leur corresponde, c'est le cas notamment pour les espaces mathématiques servant de modèle théorique à la description des entités physiques, il est par ailleurs 
difficile de s'imaginer toute forme d'appréhension des objets, aussi près soient-ils de notre perception immédiate, sans l'implication d'un certain nombre de structures géométriques formelles, qui en partie sont données apriori, et en partie dépendent peut-être de l'évolution biologique de l'organisme au cours du temps. Il apparaît clair alors qu'il faut opérer une double sinon une triple distinction. D'abord entre l'espace mathématique et l'espace sensible (ou perceptif); ensuite entre l'espace physique, qui sert de substrat aux phénomènes physiques, et l'espace sensible ; enfün, entrc l'espace mathématique et l'espace physique. Mais il faut en même temps voir qu'une telle distinction ne tient pas, d'un point de vue épistémologique, à ce que l'espace mathématique soit de nature logique et conventionnelle, l'espace physique de nature descriptive et empirique (bien que l'on admette que ses lois empiriques soient prescrites par la théorie mathématique sousjacente - il serait donc, lui aussi, en quelque sorte conventionnel), et l'espace sensible, de nature subjective. La différence ne réside pas non plus, comme l'on préfère affirmer aujourd'hui, dans le fait que les espaces mathématique et perceptif seraient de nature cognitive, alors que l'espace physique serait de nature objective, à savoir qu'il serait caractérisé aussi bien par certaines lois mathématiques formelles (par exemple, certains principes de symétrie invariants) que par des contenus physiques effectifs, relatifs aux divers modes de comportement (mécanique, cinématique, dynamique, atomique ou sub-atomique) de la matière.

D'ailleurs, la question de savoir lequel, parmi ces espaces, est celui qui satisfait le plus au concept de "spatialité naturelle", tel que défini par G. Granger, n'a pas une réponse aussi simple que l'on pourrait penser à première vue. Si par un tel concept on veut désigner le fait que l'espace est une forme a priori qui précède toute formalisation mathématique proprement dite portant sur l'espace comme concept, comme objet de pensée, et qui est done une condition nécessaire pour la perception des objets externes, alors l'espace perceptif nous apparaitra sans aucun doutc plus " naturel "que tel ou tel autre espace mathématique et même physique. L'application du concept en question soulève en fait des difficultés lorsqu'il s'agit, par exemple, de comprendre la structure géométrique qui caractérise les interactions entre certaines particules élémentaires. Nous sommes très loin dans ce cas, comme dans la plupart de ceux qu'on pourrait emprunter à la physique théorique et encore davantage aux mathématiques, de tout type de " spatialité naturelle ". Pour le moment, nous nous contenterons de faire une remarque, importante à nos yeux, et d'établir une distinction fondamentale. Chacun des trois espaces mentionnés correspond à un niveau de structure différent d'un a univers " d'objets, soit complètement idéal (virtuel), comme dans le cas des mathématiques, soit à la fois idéal et réel comme dans le cas de la physique, soit encore psycho-physique mais ayant intériorisé certains principes abstraits, comme dans le cas de la perception. À cela il faut ajouter les points suivants : 1) l'espace mathématique n'est que l'ensemble des 
propriétés et des concepts abstraits qui suffisent à le caractériser en tant qu'objet autonome, ou objet de pensée. Prenons l'exemple du concept de variété ou de celui équivalent de groupe. Il est bien connu qu'étant donné une variété, on peut y définir des "êtres "mathématiques (courbure, connexion, fibré, etc.) doués de certaines structures (analytique, différentiable, topologique, etc.), chacune étant en plus corrélée à un groupe de transformations ou à un système d'opérations. Autrement dit, l'espace est une forme de représentation d'un univers d'objets possibles (d'« idéalités " au sens de Desanti) qui, bien qu'ils aient un statut apriorique, n'en sont pas moins sujets à une genèse véritable. 2) L'espace physique correspond au fond à l'ensemble des modèles mathématiques qui sont susceptibles d'une actualisation dans un domaine précis de phénomènes physiques. Certains concepts géométriques permettent de relier directement les " êtres " mathématiques aux entités physiques. Le concept de connexion illustre de manière profonde cette actualisation de structures mathématiques idéales dans des phénomènes physiques.

\section{Sur les rapports entre l'espace et la géométrie}

Les recherches sur la géométrie intrinsèque des surfaces et des variétés à $n$ dimensions ( $n$ pouvant être très élevé mais toujours fini) susceptibles d'être plongées ou pas dans l'espace euclidien ordinaire ou dans un espace euclidien de dimension supérieure ${ }^{3}$, ont contribué à mettre en évidence les question fondamentales suivantes : à quelles conditions un espace peut être support d'une ou de plusieurs géométries? Par ailleurs, quelles sont les conditions minimales requises pour qu'une géométrie puisse donner lieu à un espace mathématiquement (et physiquement) intéressant? C'est là notre façon de reformuler le problème fondamental soulevé par Alain Michel, à savoir (dans ses propres termes) : quel jeu de propriétés opératoires d'un système abstrait se trouve être corrélatif d'une objectité spatiale? Et comment ces propriétés se trouvent-elles déterminer de manière analyticoalgébrico-géométrique des formes d'objets empiriques imaginaires? "II importe cependant de préciser que cette question est fondamentalement différente de celle qui consiste à engendrer arbitrairement des espaces par une procédure purement constructive de définitions d'entités fantasmagoriques, car, dans ce cas, on peut toujours associer à un objet mathématique donné (un groupe, par exemple) une structure algébrique supplémentaire de sorte à obtenir un objet plus complexe. Mais rien ne nous assure alors que l'objet primitif soit plus intelligible qu'il ne l'était auparavant. C'est cette conception des mathématiques comme d'un jeu formel qui inspire grosso modo la théorie des groupes finis de Lie ou la

25. Pour un examen historique approfondi de ce sujet, voir L. Boi, L. Giacardi, R. Tazzioli, $L a$ découserte de la géonétrie non euclidienne sur la pseudosphère, A. Blanchard, Paris, 1997. 
théorie des catégories. Il n'est pas sans intérêt de remarquer à ce propos que les analogies avec, notamment, la grammaire générative sont frappantes.

Mais revenons à la première question. Il s'agit d'abord d'un problème de nature purement mathématique mais dont les conséquences sont fondamentales pour une réflexion épistémologique sur l'espace, car on a là une voie d'accès privilégiée à la compréhension de ses structures en quelque sorte mathématiquement objectives, à savoir essentiellement ses propriétés locales et ses caractéristiques globales. On sait, à ce propos, que ces dernières peuvent avoir un rôle fondamental quant à la définition du type de géométrie compatible avec un espace donné. Dans cette approche, l'espace peut être philosophiquement considéré comme donné antérieurement à toute saisie formelle ou articulation à un système d'opérations abstraites (donc à une géométrie au sens mathématique du terme). Autrement dit, l'espace apparaît une sorte de continuum amorphe (de substrat continu) pourvu néanmoins d'une protogéométrie ou d'une structure mathématique primaire et, partant, apte à opposer une certaine résistance à une " constructivité * outrancière de ses objets ou à exercer des contraintes sur les possibilités de déformer ses propriétés fondamentales. Il suffit de penser respectivement au type de connexité et de singularités que peut présenter un certain espace. C'est peutêtre dans ce sens qu'A. Michel, à la suite de G. Granger, utilise la notion d'“ espace naturel ». Mais la définition, telle qu'elle a été donnée, n'était pas exempte d'une certaine ambiguïté. Selon A. Michel, "l'espace intuitif [que nous aurions introduit dans notre ouvrage pour l'opposer à la fois à l'espace mathématique et à l'espace physique] est remplacé par l'espace naturel $[\ldots]^{x}$ ». " Trois conditions sont proposées pour une pensée minimale de la spatialité : la dimensionnalité, l'opposition du local et du global, la possibilité de mesures. "On remarquera que déjà ces trois conditions font intervenir des idées mathématiques profondes qui relèvent tant de l'analyse (fonctions métriques et applications différentiables) que de la géométrie et de la topologie algébriques et différentielles. Par exemple, le concept de dimension peut avoir au moins deux sens différents : i) premièrement, celui qui sert à localiser spatialement les objets perçus du monde extérieur en les rapportant à un système abstrait de coordonnées ; $i$ ) deuxièmement, celui qui a été mis en évidence par la méthode topologique consistant à ajouter de nouvelles dimensions aux objets mathématiques comme surfaces et variétés par des coupures successives du continu spatial. On pourrait en plus mentionner une autre signification du concept de dimension, qu'on retrouve en physique et notamment dans la théorie des champs de jauge. Or, il nous semble que le concept d'espace naturel, tel qu'A. Michel et G. Granger

26. Avouons ici ne pas très bien comprendre le commentaire qui suit l'affirmation : * signe qu'on s'est écarté d'une référence à la phénoménologie, pour rapprocher le travail épistémologique de l'histoire, entendu comme analyse des ceuvres -, d'autant plus que par le concept de variété (Mannigfaltigkeit), Husserl entend précisément un domaine formel d'objets comme corrélat objectif d'un systeme formel d'axiomes. 
l'entendent, est beaucoup plus proche du premier sens que du second. Selon eux, naturel a s'oppose tout à la fois à empirique et à mathématique. Un concept naturel c'est d'abord un concept transcendantal, et ceci en un double sens. D'abord en ce qu'il détermine a priori les cadres de toute pensée d'objet. L'espace naturel est source à la fois de l'espace perceptif et de l'espace mathématique. C'est en ce sens qu'on peut dire qu'il a une naturalité anté-prédicative. Ensuite en ce qu'il est synthétique (et donc synthétique $a$ priori). Son unité synthétique provient de ce qu'il associe de façon nécessaire des propriétés non déductibles les unes des autres, qu'il réalise la synthèse entre des éléments ensuite dissociés par l'activité mathématique, aux prises avec des contraintes et des obstacles nés précisément de ce caractère synthétique, de cette solidarité des éléments constitutifs du concept, qui s'oppose à la mise en évidence de ces éléments, à la dissociation de ces concepts naturels en concepts partiels. " Or, ce serait grâce à leur caractère opératoire ou instrumental, ajoute A. Michel, que les concepts naturels réalisent une synthèse a priori des éléments mathématiques dissociés. Mais le fait d'identifier opératoire à instrumental révèle que la notion de concept naturel s'inscrit dans la conception constructiviste (ou formaliste) des mathématiques dont il a été question plus haut, plutôt que dans la démarche transcendantale qui met de l'avant le fait que les objets mathématiques idéaux seraient autant de concepts " synthétiques a priori ".

Il importe en outre de voir qu'un tel espace (l'espace ambiant) est un objet hautement virtuel, susceptible d'accueillir de nouvelles articulations et dimensions, bref de nouvelles structures. Considérons l'exemple du concept d'application (une généralisation du concept de fonction), qui est tout simplement une opération qui relie deux types d'espaces ou de variétés, disons $M^{n}$ et $M^{r}$, en se domnant deux systèmes de fonctions $\left(x_{1}, \ldots, x_{n}\right)$ et $\left(y_{1}, \ldots, y_{n}\right)$ de sorte à ce que $y_{i}=f_{i}\left(x_{1}, \ldots, x_{n}\right)$. On peut définir alors une loi qui va envoyer tout point $x$ de la variété $M^{n}$ en un point de $M^{\prime}$. Lorsque les fonctions $f_{i}$ sont différentiables avec des dérivées continues, on dit que l'application est différentiable. On la dit analytique si les fonctions sont analytiques, polynomiale si les fonctions sont polynomiales, etc. Un autre exemple significatif est celui des surfaces ou des variétés, qui peuvent admettre plusieurs niveaux de structure séparément : le métrique local et/ou global, le différentiable (de classe $C^{r}$, où $r$ n'est pas forcément infini) et le topologique (combinatoire, algébrique ou différentielle) ${ }^{D}$, ou bien les trois conjointement. Ce processus engage une recherche des " essences" des

27. À vrai dire, il faudrait en outre ajouter le niveau symplectique. Pour un exposé accessible sur ce nouveau sujet des mathématiques qu'est la géométrie et topologie symplectique, voir C. Viterbo, antroduction à la topologie symplectique *, Gazette des Mathématiciens, ${ }^{\circ} 54$. 1992. 
objets mathématiques ${ }^{3}$, et le passage d'un niveau à l'autre comporte une genèse idéale de ces mêmes objets. On peut ainsi distinguer un certain nombre de cas : i) celui où le même espace est compatible avec plusieurs géométries entre elles isomorphes; l'exemple de l'espace $R^{3}$, où l'on peut faire opérer aussi bien le groupe euclidien que le groupe non euclidien de transformations, est sans doute le plus simple; il) le cas où le même espace est compatible avec des géométries différentes ; iii) le cas où un espace donné est compatible avec une certaine géométrie mais incompatible avec une autre.

Mentionnons trois exemples précis qui illustrent bien, nous semble-t-il, les remarques précédentes. 1) Il reste à prouver que chaque surface lisse admet une immersion isométrique locale et lisse dans $R^{3}$, et qu'elle peut être plongée globalement et de façon isométrique dans $R^{4}$. Une contribution importante à la solution de ce problème a été faite pour le cas des surfaces à courbure constante négative. Par ailleurs, il a été démontré que toute métrique sur le tore plat $T^{2}$ admet une immersion isométrique dans $R^{4}$. 2) Supposons qu'une surface fermée dans $R^{3}$ armet une déformation isométrique, infinitésimale, non triviale et d'ordre quelconque ; admettra-telle aussi une déformation différentiable non triviale? Il a ćté démontré que cela est vrai si la surface et les déformations en question sont réelles et analytiques à la fois. 3) Peut-on prouver que chaque surface compacte admet un plongement isométrique dans une variété tridimensionnelle complète, simplement connexe et de courbure constante? Il est à considérer que le problème ne change pas si on considère des varićtés à trois dimensions avec des singularités isolées.

\section{Deux postulats épistémologiques sur l'espace}

1. L'espace géométrique est une construction mathématique, et une géométrie $n$ 'est, au point de vue formel et au sens moderne du terme, que la théorie des "êtres géornétriques "définis sur un certain type de variété.

Un fait qui illustre d'une façon exemplaire la signification de (i) est qu'à chaque géométrie correspond un espace et vice versa. De plus, on peut dire que dans le cadre d'une même géométrie on est libre de supposer que l'espace ait 3, 4, 5 ou $n$ dimensions ( $n$ pouvant être très élevé). Par ailleurs, tout ce qui précède représente une confirmation du fait qu'une géométrie n'est pas une construction arbitraire, ou simplement un langage formel; elle est beaucoup plus une image mentale, une idéalisation, exprimant et déterminant pour ainsi dire a priori les conditions objectives des phénomènes et les lois auxquelles obéissent les propriétés réelles des objets. L'espace mathématique n'est plus alors une forme de notre sensibilité externe au sens de Kant. On pourrait à la rigueur affirmer (avec Kant) qu'il est une intuition

28. Ces a essences " peuvent être de l'ordre de la grandeur (quantité), de la structure (relation, composition, forme), du topos (qualité, situation, déformation) et du physique (propriétés réelles\}. 
pure, mais une telle proposition n'est que partiellement vraie, car ce n'est pas l'espace comme tel qui est une intuition pure, mais plutôt la manière dont nous appréhendons l'existence de nouveaux objets mathématiques dans tel ou tel autre espace. L'espace n'est pas non plus qu'une simple forme de l'entendement au sens de Poincaré, car une fois que nous aurons conçu un monde d'entités mathématiques imaginaires, celles-ci auront une existence en quelque sorte indépendante du sujet et même, peut-être, en dehors de toute pensée : c'est bien le cas pour les entités géométriques qui existent sous la forme de phénomènes naturels. D’un point de vue général, on peut dire que l'espace est un principe d'intelligibilité, permettant d'expliquer les formes d'investissement de l'abstrait (ou formel) dans le réel. Naturellement, une telle formulation, bien que nécessaire, apparaît insuffisante dès que nous cherchons à comprendre, par exemple, la nature physique de l'espace. Il faut alors arriver à en expliquer la nature et les caractéristiques spécifiques. Une nouvelle détermination (ou des déterminations supplémentaires) est par conséquent requise. Il paraît assez évident que dans ce cas, au lieu de principe d'intelligibilité, il faudrait parler de principe constitutif ou d'engendrement, car nous n'avons plus affaire au domaine du possible (virtuel), mais à celui du réel.

2. L'espace géométrique n'est réductible ni à l'espace physique ni à l'espace physiologique. Autrement dit, l'espace géornétrique ne peut pas être formé uniquement à partir de nos sensations spatiales, car l'espace sensible n'en possède aucune des propriétés essentielles.

Comme Poincaré l'a souligné dans un célèbre texte, nous ne pouvons pas nous représenter les objets dans l'espace géométrique, mais seulement raisonner sur eux comme s'ils existaient dans cet espace. [...] Ce que nous appelons la géométrie n'est pas autre chose que l'étude des propriétés formelles d'un certain groupe continu; si bien que nous pouvons dire que l'espace est un groupe ${ }^{x}$. Nous pouvons évidemment embrasser l'idée de plusieurs espaces géométriques sans que pour cela il soit nécessaire de nous imaginer la série des sensations que nous éprouverions si nous vivions dans un de ces espaces. Cette dernière remarque nous permet de passer à quelques considérations sur le conventionnalisme géométrique de Poincaré.

\section{la signification épistémologique du concept de goupe et le niveau structural des "ètres " mathématiques}

Le concept de groupe continu de transformations a joué un rôle mathématique et épistémologique considérable dans l'œuvre de Poincaré, notamment en tout ce qui a trait aux fondements de la géométrie et à la théorie de l'espace. Le concept de structure de groupe y apparaît d'ailleurs tout à fait fondamental. En fait, il se trouve au cour même de la conception que Poincaré a des mathématiques. Il s'agit pour lui d'un concept intra-

29. Voir H. Poincaré, Des fondements de La géométrie, Chiron, Paris, 1921, p. 11. 
mathématique ; il est, pour ainsi dire, un concept générateur à partir duquel il est possible d'engendrer formellement de nouveaux objets mathématiques. Bref, celui de groupe est à la fois un concept formel et constitutif des mathématiques. Poincaré a affirmé (nous citons librement) que toute théorie mathématique est, en dernière analyse, l'étude des propriétés d'un groupe d'opérations, c'est-à-dire d'un système formé par certaines opérations fondamentales et par toutes les combinaisons qu'on peut en faire. Si, dans une autre théorie, on étudie d'autres opérations qui se combinent d'après les mêmes lois, celle-ci contiendra une suite de théorèmes qui peuvent être mis en correspondance bijective avec ceux de la première théorie, et les deux théories pourront se développer avec un parallélisme parfait. On dit alors que les deux groupes d'opérations sont isomorphes ou bien qu'ils ont la mème structure. Si alors on dépouille la théorie mathématique de ce qui n'y apparaît que comme un accident, c'est-à-dire de sa matière, il ne restera que l'essentiel, c'est-à-dire la forme, qui constitue pour ainsi dire le squelette solide de la théorie ; ce sera la structure du groupe.

Les propriétés formelles sont donc celles qui sont communes à tous les groupes isomorphes. Poincaré distingue entre l'ordre et le degré d'un groupe. Il appelle le degré du groupe le nombre des objets (ou des éléments d'une figure), et l'ordre le nombre des permutations dont ces objets sont susceptibles. Or, deux groupes isomorphes auront toujours le même ordre. D'après lui, le degré est donc un élément matériel pour ainsi dire, et l'ordre, un élément formel dont l'importance est beaucoup plus grande. Dans le cas des groupes continus, on suppose généralement que l'objet des opérations du groupe est un ensemble de $n$ quantités, appelées coordonnées susceptibles de varier d'une manière continue. Aux yeux de Poincaré, les propriétés formelles d'un groupe non seulement sont importantes sur le plan mathématique abstrait, mais également d'un point de vue épistémologique. Voici quelques-unes des propriétés formelles fondamentales d'un groupe.

1) Un groupe est continu; ce qui revient à dire qu'un déplacement quelconque peut être divisé un nombre infini de fois, c'est-à-dire qu'on peut toujours trouver un autre déplacement qui, répété un certain nombre de fois, reproduira un déplacement donné. Cette divisibilité à l'infini nous conduit, d'après Poincaré, à la notion de continuité mathématique.

2) Un groupe admet l'existence de sous-groupes. Ce fait n'est pas du tout arbitraire : on ne peut pas former autant de sous-groupes qu'on veut. En effet, un résultat fondamental de la théorie des groupes de Lie dit que les groupes se distinguent les uns des autres, au point de vue formel, par le nombre de sous-groupes qu'ils admettent et par les relations qui existent entre eux. Or, il est un fait de la plus grande importance que les transformations infinitésimales du même sous-groupe soient identiques, et que les transformations d'un sous-groupe donné soient identiques les unes aux autres et identiques au sous-groupe primitif. On dit alors que ce sous- 
groupe est invariant. Il est clair par ailleurs que la géométrie possédant le nombre le plus grand de sous-groupes sera à considérer la plus riche, c'est-àdire celle dotée d'un degré de générativité plus élevé.

Le caractère formel du concept de groupe tient à ce qu'il permet de découvrir un niveau supérieur d'existence des " êtres " mathématiques (une existence d'un autre ordre) : celui de structure. Les notions et théories mathématiques peuvent ainsi être tout à fait distinctes quant à leur's « objets " propres, si bien qu'elles soient identiques au point de vue de leurs structures. C'est là un caractère essentiel des mathématiques que Poincaré s'efforce de mettre en évidence tout au long de son œuvre. En effet, à toute théorie mathématique correspond un certain groupe de transformations s'appliquant aux éléments de la théorie, et l'ensemble de ces transformations ou les opérations suivant lesquelles on fait opérer un groupe sur un objet mathématique donné - qu'il soit une figure géométrique simple, un espace, ou une fonction - constituent ce qu'on appelle la structure du groupe. La structure représente pour ainsi dire le « noyau » de chaque groupe : en effet, on peut faire varier les éléments qui le composent, alors que les propriétés essentielles de la figure sur laquelle le groupe opère se conservent; autrement dit, ces propriétés sont invariantes vis-à-vis du groupe fondamental de transformations. Évidemment un tel fait n'est pas arbitraire, car les mêmes propriétés caractérisant une figure géométrique (ou objet mathématique) domnée peuvent être invariantes par rapport à un certain groupe de transformations et ne plus l'être par rapport à un autre groupe. On pourrait dire que la structure du groupe exprime "la permanence dans la variation „ de l'ensemble des propriétés appartenant à cette même figure géométrique (ou à ce même objet mathématique) : on peut notamment substituer les éléments d'une figure par ceux d'une autre, sans que les transformations auxquelles ils sont assujettis altèrent leurs propriétés et relations caractéristiques.

Or, c'est bien pour cet ensemble de raisons que Poincaré attribue autant d'importance au concept de groupe dans toutes les mathématiques sans exception, et en particulier à la notion d" isomorphisme "qui lui est intimement liée : deux groupes différents par leur nature, mais identiques au point de vue de leurs lois (formelles), sont dits être isomorphes. Par exemple, le groupe des mouvements non euclidiens du cercle fondamental (ou plan non euclidien) est identique à celui des mouvements euclidiens du plan euclidien ; cette identité concerne les opérations formelles (les structures) de ces groupes, mais non pas la forme concrète des figures correspondantes, c'est-à-dire le cercle fondamental et le plan euclidien. De l'isomorphisme entre des groupes découle celui plus général entre deux ou plusieurs théories mathématiques ou entre une théorie mathématique et une théorie physique qui, bien qu'étant différentes quant à la nature de leurs objets, sont équivalentes relativement à la structure de leurs groupes respectifs. C'est ainsi - remarque Poincaré - que la géométrie non euclidienne à trois 
dimensions de Lobatchevsky est équivalente à la géométrie projective des figures, réelles ou imaginaires, qui existent sur une droite. C'est ainsi encore qu'un "événement " dans la cinématique de Lorentz correspond, dans la géométrie de Laguerre, à une sphère orientée; un rayon lumineux se propageant dans le vide correspond à un faisceau de sphères orientées tangentes.

Les exemples de correspondance (ou d'équivalence) en termes de structure géométrique entre théories mathématiques et théories physiques sont de nos jours encore plus frappantes et ce, en dépit des difficultés parfois considérables qui s'opposent à une connaissance des structures de groupe d'un bon nombre de classes de phénomènes physiques, en particulier du monde microscopique. Ces difficultés sont peut-être en partie dues à la complexité de leur géométrie, et au fait qu'on ne possède pas, dans certains cas, des critères d'individuation les plus primaires pour permettre une classification des propriétés structurales communes à ces diverses classes de phénomènes. La question se pose toujours, par exemple, de savoir quels sont les groupes (ou semi-groupes) qui correspondent aux états solide, liquide et gazeux de la matière. Poincaré avait la conviction intime que toute théorie physique, qu'elle fût de nature mécanique ou dynamique, devait se fonder sur une structure mathématique relativement simple et unitaire, c'est-à-dire sur un groupe de transformations opérant de façon covariante sur l'espace des phénomènes physiques considéré (c'est-à-dire l'espace de phase). Cette recherche l'a conduit, entre autres, à énoncer mathématiquement les lois invariantes du groupe de Lorentz auxquelles obéissent les phénomènes électromagnétiques et gravitationnels, et par là à jeter les bases du postulat de la relativité restreinte. Ce qui précède permet de tirer une première conclusion : l'existence d'un isomorphisme entre divers groupes, et entre différentes théories mathématiques ou physiques, constitue la véritable racine du conventionnalisme géométrique de Poincaré.

\section{Essai d'une nouvelle interprétation du conventionnalisme géométrique}

Faisons d'abord trois remarques préliminaires.

(i) L'interprétation " standard " (ou néo-empiriste) du conventionnalisme consiste à séparer la géométrie théorique, vue comme un ensemble de théories axiomatiques formelles, de la géométrie appliquée, qui aurait comme fonction de donner une interprétation physique appropriée d'une de ces théories axiomatiques. Suivant ce schéma, donc, le caractère conventionnel de la géométrie appliquée découle nécessairement du caractère conventionnel de la géométrie théorique. Selon ce point de vue, que l'on peut qualifier de " linguistique ", la géométrie théorique est conventionnelle en vertu du " principe d'intertraductibilité ", selon lequel toute théorie axiomatique peut en principe être traduite dans une autre. 
(ii) La façon dont Poincaré conçoit le rapport entre la géométrie et l'expérience peut être résumée ainsi : bien que notre construction de l'espace géométrique soit suggérée et même guidée par certaines expériences primaires relevant de l'espace intuitif, il n'en est pas moins vrai qu'une fois que nous avons formé le concept d'espace géométrique, la géométrie devient apte à décrire tout fait physique d'une nature spatiale quelconque, et bien sûr même ceux qui n'ont eu aucun rôle direct dans la constitution de l'espace géométrique.

(iii) La critique de l'empirisme faite par Poincaré s'appuie sur deux sortes d'arguments : premièrement, sur un argument de principe, selon lequel la géométrie ne peut être une science empirique, car elle ne peut être soumise à une vérification expérimentale; deuxièmement, sur un argument théorique général qui dit que, à la différence des sciences naturelles, la géométrie est une science exacte : elle ne cherche pas à décrire la réalité telle qu'elle est ou telle qu'elle nous apparait, mais à la " construire " au moyen de principes et d'hypothèses théoriques; elle ne s'intéresse pas à savoir si ses objets correspondent ou non aux objets existant dans la réalité, mais plutôt à les idéaliser (éventuellement à les formaliser) et à en donner une explication théorique.

La nature du conventionnalisme géométrique de Poincaré tient au fond à une compréhension géométrique des phénomènes. Autrement dit, le conventionnalisme fournit les concepts nécessaires permettant de relier l'analyse à la physique. Si ces concepts sont des conventions, ce n'est pas parce que leur contenu est purement linguistique ou parce qu'ils rendent possible une application simple des théories mathématiques abstraites à la physique " concrète ", mais plutôt parce qu'ils sont des idéalisations des phénomènes, des images ou des formes possibles de leurs structures. Il nous semble en ce sens difficile d'accepter la conception selon laquelle la signification de la géométrie reviendrait en fait à postuler l'institution d'un système de propositions servant uniquement à choisir de façon arbitraire un étalon de mesure ou un ensemble de lois " coordonnatrices ". Ces dernières, cependant, bien que n'ayant d'autre sens que celui qui leur est attribué par la géométrie elle-même, auraient en définitive comme fonction de soumettre ces propositions à une vérification expérimentale. C'est la conception, entre autres, de Hans Reichenbach. C'est dire que la géométrie ne serait au fond qu'un " système de repère " auquel sont ramenées ou au moyen duquel sont comparées les entités physiques. Ainsi, ces dernières sont susceptibles d'une détermination véritable parce qu'elles peuvent être mesurées ; c'est donc grâce à l'opération de mesure qu'elles deviennent parfaitement compréhensibles dans le cadre d'une théorie : il s'agit bien d'un point de vue "opérationnaliste ". Cette position a prétendu s'inspirer des idées de Poincaré sur le statut de la géométrie, et en particulier de l'idée qu'une géométrie ne peut pas être expérimentalement plus " vraie " qu'une autre, car 
il peut y avoir plusieurs géométries différentes aptes à expliquer les mêmes phénomènes physiques.

Cependant, à vouloir approfondir cette question, on est forcé d'admettre que le conventionnalisme de Poincaré a peu de points en commun avec le néo-empirisme ou l'a opérationnalisme ». En réalité, il tient au fait essentiel qu'il existe un écart inéluctable entre la théorie et l'expérience et entre la géométrie et la physique proprement dite. La découverte des géométries non euclidiennes et d'autres théories mathématiques, notamment la théorie des groupes de transformations par Sophus Lie, a mis en évidence qu'en tant que théorie mathématique, la géométrie n'est plus une image unique du monde physique. Cette découverte a de plus servi à montrer qu'étant donné une certaine classe de phénomènes physiques, il est possible d'en fournir diverses interprétations géométriques, lesquelles, dans un certain sens, sont comparables entre elles mais non forcément toutes également valables. Déjà Lobatchevsky avait compris ce fait fondamental. Bien qu'il fût convaincu du fait que les phénomènes physiques, dans les limites de nos moyens d'observation et de nos expériences, s'accordent à peu près aux lois de la géométrie euclidienne, il n'a pas écarté pour autant la possibilité que certaines forces de la nature suivent une géométrie et d'autres, leur géométrie propre. Peut-être cette géométrie est-elle celle des forces moléculaires ${ }^{30}$... Gauss de son côté, encore plus explicitement que Lobatchevsky, avait reconnu qu'il suffisait de supposer la constante $K$ (c'està-dire la courbure de l'espace) infiniment grande pour admettre l'existence de phénomènes physiques qui auraient des propriétés géométriques différentes de celles admises par la géométrie euclidienne. Riemamn, comme on le sait, avait poussé beaucoup plus loin que ses prédécesseurs la réflexion sur cette question, en arrivant à la conclusion que les phénomènes physiques dans l'infiniment petit pourraient être régis par une géométrie non euclidienne. Quant à Poincaré, il se limite d'abord et avant tout à considérer l'aspect mathématique de cette transformation conceptuelle du statut de la géométrie et du concept d'espace. Plus précisément, il souligne le fait qu'il est possible d'obtenir plusieurs géométries à partir d'un petit nombre d'objets mathématiques et de leurs propriétés caractéristiques; ces géométries peuvent être en outre équivalentes.

Ce qui précède a été mis en évidence, de différentes manières, par le développement de la géométrie au XIX ${ }^{e}$ siècle, et en particulier : (i) par l'idée de Riemann qu'aux mêmes rapports d'étendue peuvent correspondre différents rapports métriques (c'est-à-dire des formes quadratiques et même non quadratiques - des formes différentielles plus générales - aux propriétés mathématiques très diverses); (ii) par le résultat dù à Beltrami

30. François Lalonde a attiré à nouveau notre attention sur ce passage du géomètre russe ainsi que sur ceux de Riemann où se trouve précisée une telle idée. L'affinité avec certaines conceptions récentes concernant le type de structure géométrique qui régirait l'infiniment petit est tout à fait frappante. 
montrant qu'il est possible de fournir un modèle euclidien de la géométrie non euclidienne plane de Lobatchevsky. Ce résultat de Beltrami conduit à l'idée beaucoup plus générale qu'il est possible de construire plusieurs modèles d'une théorie mathématique dans le cadre d'une même théorie, pourvu que l'on dispose d'une théorie mathématique de base, moyen terme reliant les deux autres théories ; (iii) grâce à la conception de Lie et de Klein, selon laquelle les propriétés essentielles d'une géométrie peuvent être exprimées au moyen d'un groupe de transformations, vis-à-vis desquelles elles demeurent invariantes. De ce point de vue, chaque géométrie se distinguera des autres parce qu'elle admet un certain groupe de transformations : l'ensemble des déplacements dans l'espace usuel formant le groupe de transformations de la géométrie euclidienne en est le plus élémentaire.

Or, ce sont bien là les vraies origines du conventionnalisme géométrique de Poincaré. Il le voyait comme une position intermédiaire entre l'empirisme et l'apriorisme. Poincaré justifie son point de vue de la manière suivante : mathématiquement, la géométrie n'est que l'étude d'un groupe de transformations donné, et puisqu'il y a des groupes qui sont isomorphes, les géométries auxquelles ils correspondent doivent l'être aussi, pourvu que certaines conditions soient satisfaites. Par conséquent, on ne peut affirmer que la géométrie d'Euclide et la géométrie de Lobatchevsky, par exemple, soient mathématiquement incompatibles ; les résultats de Riemann, de Beltrami, de Klein et de Poincaré lui-même sont là pour prouver le contraire. On ne peut pas non plus affirmer d'une manière absolue qu'une géométrie soit plus vraie qu'une autre. Un deuxième aspect de la question, concernant le rapport entre la géométrie et la physique, doit cependant être évoqué ici. À ce propos, la nature du groupe n'est pas indifférente quant au fait de savoir si une géométrie peut expliquer les phénomènes physiques mieux qu'une autre, car, en effet, à tout espace physique se trouve intrinsèquement associé un groupe de transformations géométriques laissant invariantes toutes les lois de la nature. La position conventionnaliste de Poincaré sur la géométrie théorique devrait ainsi être complétée par une position « réaliste " relative au problème du choix du groupe utile à l'interprétation des phénomènes physiques. La réponse de Poincaré a consisté dans la mise en évidence de deux critères : la simplicité mathématique du groupe, et le fait qu'il soit apte à représenter mieux qu'un autre les mouvements des corps solides. En effet, les divers mouvements de ces corps doivent avoir les mêmes relations que les diverses opérations du groupe.

Mais du point de vue de la physique mathématique actuelle, la réponse de Poincaré apparaît plutôt insatisfaisante, alors que les idées développées par Élie Cartan et Hermann Weyl autour des années trente et quarante de ce siècle se sont révélées beaucoup plus fécondes pour comprendre les rapports entre la géométrie et la physique. Ainsi, la conception philosophique que Poincaré a de la géométrie doit être révisée et complétée par les deux points 
suivants : a) l'existence de plusieurs géométries toutes mathématiquement valables n'exclut pas que l'une d'elles soit plus apte qu'une autre à représenter et à expliquer une certaine classe de phénomènes ; $b$ ) ces géométries n'ont pas forcément le même statut et la même validité mathématiques ; il existe en fait des critères intrinsèques de discrimination permettant d'adopter une géométrie plutôt qu'une autre.

Revenons, pour conclure, sur l'affirmation de tout à l'heure : le conventionnalisme géométrique de Poincaré se fonde sur le nouveau rôle que joue le concept de groupe en mathématiques. L’importance épistémologique de ce concept devrait pouvoir ressortir des considérations suivantes.

(i) Le concept de groupe est un concept intra-mathématique, dans le sens qu'il est un principe commun à l'ensemble des disciplines mathématiques ; il a par ailleurs permis d'élargir considérablement l'étude d'objets et de structures mathématiques en partie déjà connues et d'en découvrir de nouvelles.

(ii) Il a permis de découvrir des analogies profondes entre des théories mathématiques considérées encore à la fin du XIX $\mathrm{X}^{\mathbf{e}}$ siècle comme étant tout à fait distinctes (un exemple important est celui de la découverte, faite par Poincaré lui-même, de l'analogie entre la géométrie non euclidienne de Lobatchevsky et la théorie des fonctions automorphes), ainsi que des affinités entre des théories mathématiques et des théories physiques.

(iii) Il est au cour d'un des aspects les plus importants du développement des mathématiques modernes : le passage du local au global et le fait que beaucoup de problèmes mathématiques ont une nature géométrique globale.

(iv) Son application à diverses branches des mathématiques et de la physique a contribué à dégager une conception structurale de la science, tout en ayant montré en même temps le rôle primordial que joue l'intuition géométrique dans la découverte mathématique et dans le développement de nouvelles méthodes. En fait, l'intuition est l'acte de donner une forme géométrique à un " objet "mathématique (abstrait) quelconque. Cela montre que le statut de l'intuition en mathématiques est étroitement apparenté à l'émergence d'une conception qualitative.

Certes, par l'élaboration de son conventionnalisme géométrique, Poincaré a voulu exprimer phílosophiquement l'importance du rôle pris par la notion de groupe de transformations et de la théorie des invariants, sur lesquelles est fondée une partie fondamentale des mathématiques et de la physique modernes. Le problème reste à savoir s'il y est vraiment parvenu. Les dernières remarques visaient, entre autres, à montrer que sa conception épistémologique concernant le statut de la géométrie ainsi que la nature à attribuer à la géométrie physique apparaît profondément insatisfaisante et ce, 
surtout à la lumière des développements récents dans la géométrisation des mathématiques et de la physique théorique, et qu'elle exige donc une révision substantielle.

Département de philosophie

Université du Québec à Montréal et

Institute for Advanced Studies, Princeton 